\title{
Multiscale Modeling of Failure in Composites under Model Parameter Uncertainty
}

\author{
Michael J. Bogdanor ${ }^{\dagger}$, Caglar Oskay ${ }^{\dagger *}$ and Stephen B. Clay ${ }^{\ddagger}$ \\ ${ }^{\dagger}$ Department of Civil and Environmental Engineering, \\ Vanderbilt University, Nashville, TN 37235, USA \\ ${ }^{\ddagger}$ Aerospace Systems Directorate, Air Force Research Laboratory, \\ Wright-Patterson Air Force Base, OH 45433, USA
}

\begin{abstract}
This manuscript presents a multiscale stochastic failure modeling approach for fiber reinforced composites. A homogenization based reduced-order multiscale computational model is employed to predict the progressive damage accumulation and failure in the composite. Uncertainty in the composite response is modeled at the scale of the microstructure by considering the constituent material (i.e., matrix and fiber) parameters governing the evolution of damage as random variables. Through the use of the multiscale model, randomness at the constituent scale is propagated to the scale of the composite laminate. The probability distributions of the underlying material parameters are calibrated from unidirectional composite experiments using a Bayesian statistical approach. The calibrated multiscale model is exercised to predict the ultimate tensile strength of quasi-isotropic open-hole composite specimens at various loading rates. The effect of random spatial distribution of constituent material properties on the composite response is investigated.
\end{abstract}

Keywords: fiber reinforced composites, stochastic failure prediction, multiscale models, random material properties, spatial variability

\section{Introduction}

Failure prediction of composite materials is an inherently multiscale problem from the mechanistic and probabilistic standpoints. The physical mechanisms that govern failure in composites operate at multiple spatial scales. For instance, diffuse damage in the form of microcracks and voids incubates at the scale of the material microstructure, grows and coalesces at the scale of the mesostructure

\footnotetext{
${ }^{*}$ Corresponding author. Address: VU Station B\#351831, 2301 Vanderbilt Place, Nashville, TN 37235, United States
} 
(i.e., characteristic volume), and ultimately leads to failure at the scale of the macrostructure (i.e., a coupon or component). An illustration of the size scales in fiber reinforced composites is given in Fig. 1. Natural material variability and manufacturing process induced defects at each size scale cause composites to exhibit significant variations in structural strength, failure response and other mechanical properties. Defects within the composite constituents (i.e., matrix, fiber) at the scale of the material microstructure; variability in the fiber volume ratio, void distribution, and residual stresses at the scale of the characteristic volume; and fiber misalignment, thickness variability and interlaminar defects at the scale of a ply all lead to the property and behavior uncertainty observed at the scale of the macrostructure. Accurate and reliable prediction of the failure of composites is therefore critically linked to the incorporation of uncertainty at the pertinent length scales.

A number of approaches have been proposed in the literature for the incorporation and propagation of uncertainty in composites across spatial scales. A comprehensive overview of the literature in this area was presented by Sriramula and Chryssanthopoulos in Ref. [37]. The effect of random constituent elastic properties on the effective composite elastic moduli has been investigated by Kaminski [20] and Sakata, et al. [28] using the stochastic finite element method (SFEM). Shaw and coworkers [32] estimated effective composite properties based on random constituent strength and stiffness using Monte Carlo (MC) simulation and applied first and second order reliability methods (FORM and SORM) to predict the failure of a laminated plate based on the Tsai-Hill failure criterion. Reliability of laminated composites based on random composite properties and layer thicknesses was investigated using SFEM and MC, $\beta$, and first order second moment reliability methods [22], the Edgeworth expansion method [21], and FORM and MC with trained neural networks [23]. The propagation of uncertainty from random constituents up through the effective composite properties to compute the reliability of composite structures using perturbation based stochastic finite elements has been investigated in Refs. [33, 5]. More recently, a Bayesian statistical approach was proposed to perform calibration of constituent material properties at the scale of the characteristic volume of a unidirectional ply based on ply-level experimental data [4].

The variability in the composite response can be linked to uncertainty at its microstructure using multiscale computational methods such as the $\Delta$-criterion [16], the non-concurrent multiscale stochastic method [7], and homogenization-based methods such as stochastic finite elements [20], perturbation-based homogenization [30], and the multiscale spectral stochastic method [38], among others. Computational homogenization provides a rigorous basis for linking the fine and coarse scale response of composites and other heterogeneous materials, and has been successfully employed to compute effective elastic properties and failure in a deterministic setting (see e.g., [17, 9, 14, 19, 40]). While the extensions of computational homogenization to compute the variability in homogenized elastic properties from known variability at the scale of the microstructure to study stochastic problems has been proposed [20,30,38], the application of this approach to study the uncertainty in the inelastic and failure properties has proven more difficult. The difficulty is largely computational 


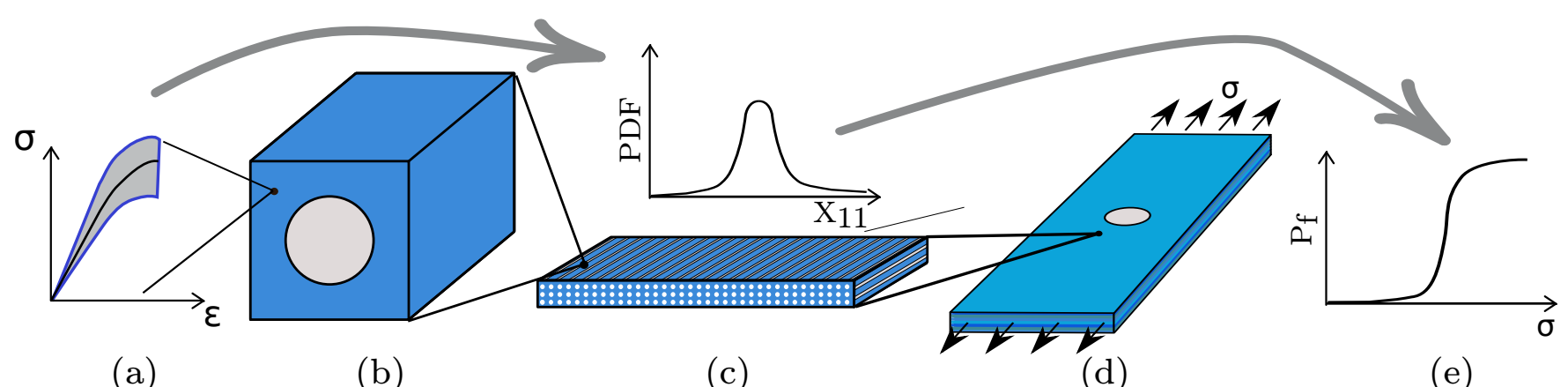

(a)

(b)

(c)

Figure 1: Multiple scales in a fiber reinforced composite material: (a) constituent, (b) characteristic volume, (c) ply with random strength distribution, (d) component, (e) failure probability of a component.

- additional dimensions introduced into the problem by the random treatment of microstructural material and morphological parameters lead to computational intractability when the material response includes damage and nonlinearity. As Fish and $\mathrm{Wu}[13]$ recently demonstrated, model order reduction approaches for the multiscale solvers e.g., [8, 24, 41], as well as efficient uncertainty quantification algorithms $[15,39,6]$ are critical to the development of computationally tractable stochastic multiscale modeling of composite materials.

In this manuscript, a new probabilistic multiscale methodology is developed to link the material property variability at the scale of the microstructure to the laminate scale and predict the uncertainty associated with the composite strength. The proposed methodology relies on a reduced order computational homogenization model to bridge the macro- and the microscales. Bayesian statistical analysis with Markov chain Monte Carlo (MCMC) sampling forms the foundation of the uncertainty quantification framework. Gaussian process modeling is used to approximate the failure response of the multiscale simulations in the rapid sampling MCMC process. The uncertainty at the scale of the microstructure is modeled as random constituent material parameters governing the evolution of damage and failure in the distinct microscopic phases. The proposed probabilistic multiscale approach is applied to investigate the rate-dependent response of carbon fiber reinforced composite laminates. The microscale failure properties and their variability are calibrated based on ply-level experimental data. The calibrated model is validated against separate experimental measurements of the ultimate tensile strength of quasi-isotropic open-hole composite specimens at various loading rates. The effect of macroscopic spatial distribution of the constituent failure properties on the composite response is investigated through a parametric analysis. This manuscript: (1) provides a new method to propagate uncertainty from the characteristic volume of a heterogeneous material to the macroscopic scale in the context of computational homogenization; (2) employs the Bayesian statistical method to stochastic constituent parameter calibration in composites; and (3) characterizes the influence of macroscopic spatial parameter variability on the failure response of composite 


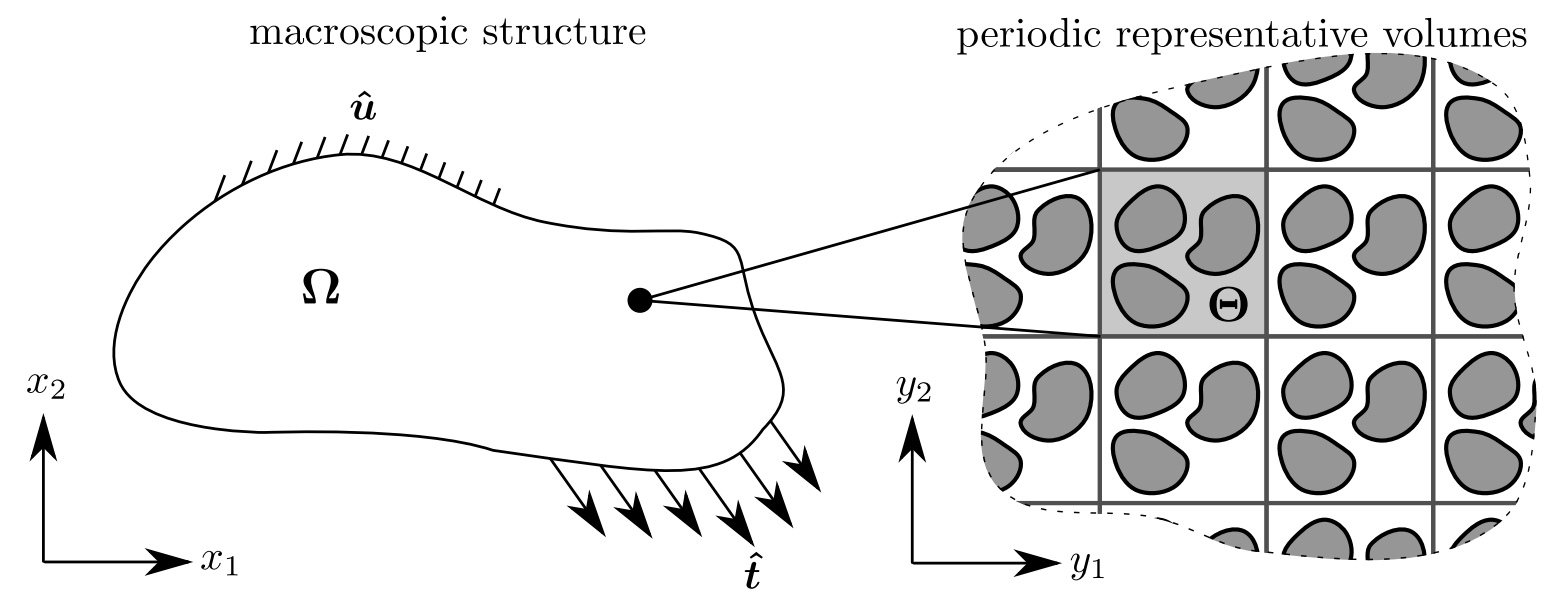

Figure 2: Schematic illustration of the composite volume.

laminates.

The remainder of this manuscript is organized as follows: in Section 2 the multiscale model for predicting the damage evolution in composites is presented. Uncertainty quantification and the Bayesian parameter updating methodology are described in Section 3. Section 4 includes a description of the suite of experiments on carbon fiber reinforced polymer composites and shows the results of calibration and validation of the multiscale model. A parametric study on the effect of macroscopic spatial variability of the constituent properties on the overall failure of a laminated composite specimen at the macroscale is also included. The conclusions and future research directions are discussed in Section 5.

\section{Multiscale Composite Damage Model}

Consider a macroscopic domain of interest, $\Omega \subset \mathbb{R}^{d}(d=2$ or 3 indicates the spatial dimensions), made of a heterogeneous, periodically repeating characteristic volume, $\Theta \subset \mathbb{R}^{d}$. The characteristic volume consists of two or more constituent material phases. This multiscale structure is schematically illustrated in Fig. 2. The failure response of the structure under quasi-static conditions is governed by the following equations:

$$
\begin{gathered}
\nabla \cdot \boldsymbol{\sigma}(\mathbf{x}, t)+\mathbf{b}(\mathbf{x})=0 \\
\boldsymbol{\sigma}(\mathbf{x}, t)=[1-\omega(\mathbf{x}, t)] \mathbf{L}(\mathbf{x}): \boldsymbol{\epsilon}(\mathbf{x}, t)=\mathbf{L}(\mathbf{x}):[\boldsymbol{\epsilon}(\mathbf{x}, t)-\boldsymbol{\mu}(\mathbf{x}, t)] \\
\boldsymbol{\epsilon}(\mathbf{x}, t)=\nabla^{s} \mathbf{u}(\mathbf{x}, t)
\end{gathered}
$$

where $\mathbf{u}$ is the displacement field, $\boldsymbol{\sigma}$ the Cauchy stress, $\mathbf{b}$ the body force, $\boldsymbol{\omega}$ the scalar damage variable such that $\omega \in[0,1), \mathbf{L}$ the tensor of elastic moduli, $\boldsymbol{\epsilon}$ the total strain, and $\boldsymbol{\mu}=\omega \boldsymbol{\epsilon}$ 
the inelastic strain. $\nabla \cdot(\cdot), \nabla(\cdot)$, and $\nabla^{s}(\cdot)$ are the divergence, gradient, and symmetric gradient operators, respectively. Equation 2 implies a damage-elastic constitutive behavior for the composite constituents. In the functional form,

$$
\dot{\omega}(\mathbf{x}, t)=f_{\omega}(\boldsymbol{\sigma}, \boldsymbol{\epsilon}, \mathbf{s} ; \boldsymbol{\psi})
$$

where the superimposed dot indicates the derivative with respect to time, $\mathbf{s}$ is the set of internal state variables, which dictate the history dependence of damage evolution, and $\boldsymbol{\psi}$ are the material constitutive parameters, which govern the damage evolution law. The particular form of the damage evolution equations for the growth of damage and additional state variables is shown in Section 2.2. The damage evolution parameters, $\boldsymbol{\psi}$, are subject to variability, which reflects the initial defect distribution within the constituent materials.

The macroscale domain is subjected to the following boundary conditions:

$$
\begin{aligned}
\mathbf{u}(\mathbf{x}, t) & =\hat{\mathbf{u}}(\mathbf{x}, t) ; \quad \mathbf{x} \in \Gamma_{\mathrm{u}} ; \quad t \in\left[0, t_{f}\right] \\
\boldsymbol{\sigma} \cdot \mathbf{n} & =\hat{\mathbf{t}}(\mathbf{x}, t) ; \quad \mathbf{x} \in \Gamma_{\mathrm{t}} ; \quad t \in\left[0, t_{f}\right]
\end{aligned}
$$

where $\hat{\mathbf{u}}$ and $\hat{\mathbf{t}}$ are the prescribed displacements and tractions on the boundaries $\Gamma_{\mathrm{u}}$ and $\Gamma_{\mathrm{t}}$ with $\Gamma=\Gamma_{\mathrm{u}} \cup \Gamma_{\mathrm{t}}$ and $\Gamma_{\mathrm{u}} \cap \Gamma_{\mathrm{t}}=\emptyset$, and $\mathbf{n}$ is the unit normal to $\Gamma$; $t_{f}$ is the time to failure. In this setting, time is employed to account for the viscous (rate-dependent) behavior of the material under quasi-static loading. Inertial effects are taken to be small and are not included.

The response fields fluctuate at the scale of the microstructure due to the heterogeneity of the constituent materials in $\Theta$. The scale of material heterogeneity is captured by introducing a scaled coordinate system, $\mathbf{y}=\mathbf{x} / \zeta$, in which $0<\zeta \ll 1$ is the scaling parameter. The coordinates $\mathbf{y}$ are employed to parameterize the characteristic volume, $\Theta$. The response fields are written in terms of both macroscale and microscale coordinates as:

$$
\varphi(\mathbf{x}, t)=\hat{\varphi}(\mathbf{x}, \mathbf{y}(\mathbf{x}), t)
$$

in which $\varphi$ denotes an arbitrary response field. The displacement field is decomposed through a two-scale asymptotic expansion with contributions from micro- and macroscopic scales as:

$$
\mathbf{u}(\mathbf{x}, \mathbf{y}, t)=\overline{\mathbf{u}}(\mathbf{x}, t)+\zeta \mathbf{u}^{1}(\mathbf{x}, \mathbf{y}, t)
$$

where $\overline{\mathbf{u}}$ is the macroscopic displacement field and $\mathbf{u}^{1}$ is the microscopic displacement field. Asymptotic expansion of the displacements allows the micro- and macroscale problems to be posed in a separate, but coupled manner.

The overall strain field contains contributions from the macroscale strain state and the locally 
fluctuating microscale strains,

$$
\boldsymbol{\epsilon}(\mathbf{x}, \mathbf{y}, t)=\overline{\boldsymbol{\epsilon}}(\mathbf{x}, t)+\nabla_{y}^{s} \mathbf{u}^{1}(\mathbf{x}, \mathbf{y}, t)
$$

where $\nabla_{y}^{s}$ denotes the symmetric gradient operator with respect to the microscopic coordinates and $\overline{\boldsymbol{\epsilon}}$ is the macroscale strain obtained by spatial averaging of the strain field:

$$
\overline{\boldsymbol{\epsilon}}(\mathbf{x}, t)=\frac{1}{|\Theta|} \int_{\Theta} \boldsymbol{\epsilon}(\mathbf{x}, \mathbf{y}, t) d \mathbf{y}
$$

in which $|\Theta|$ is the volume of the characteristic volume element. Equation 10 is a consequence of the periodicity of the response fields (e.g. $\mathbf{u}^{1}$ ) over the boundaries of the microstructural domain, $\Theta$. Using the damage-elastic constitutive law, the stress in the microscale is given as

$$
\boldsymbol{\sigma}(\mathbf{x}, \mathbf{y}, t)=\mathbf{L}(\mathbf{y}):\left[\overline{\boldsymbol{\epsilon}}(\mathbf{x}, t)+\nabla_{y}^{s} \mathbf{u}^{1}(\mathbf{x}, \mathbf{y}, t)-\boldsymbol{\mu}(\mathbf{x}, \mathbf{y}, t)\right]
$$

in which the tensor of elastic moduli is taken to vary as a function of the microscopic coordinate only, $\boldsymbol{\mu}$ are the inelastic strains in the microstructure due to damage, and the homogenized macroscale stress is expressed as:

$$
\overline{\boldsymbol{\sigma}}(\mathbf{x}, t)=\frac{1}{|\Theta|} \int_{\Theta} \boldsymbol{\sigma}(\mathbf{x}, \mathbf{y}, t) d \mathbf{y} .
$$

It is natural to consider the elastic moduli tensor of the constituent materials as random variables due to the presence of manufacturing induced defects in a composite microstructure. The effect of variability in elastic moduli of constituents on effective composite properties has been established in a number of publications (e.g. Refs. [5, 12, 20, 29, 32]). The focus of the present effort is on failure parameters only and moduli are set as deterministic.

Substituting the displacement decomposition and strain and stress expressions into the equilibrium equation (Eq. 1), along with the scaling relationship, the equilibrium equations at $\mathcal{O}\left(\zeta^{-1}\right)$ and $\mathcal{O}(1)$ are obtained:

$$
\begin{aligned}
\mathcal{O}\left(\zeta^{-1}\right): & \nabla_{y} \cdot\left[\mathbf{L}(\mathbf{x}, \mathbf{y}):\left[\overline{\boldsymbol{\epsilon}}(\mathbf{x}, t)+\nabla_{y} \mathbf{u}^{1}(\mathbf{x}, \mathbf{y}, t)-\boldsymbol{\mu}(\mathbf{x}, \mathbf{y}, t)\right]\right]=0 \\
\mathcal{O}(1): & \nabla_{x} \cdot \boldsymbol{\sigma}(\mathbf{x}, \mathbf{y}, t)=0
\end{aligned}
$$

Equation 13 constitutes the microscale equilibrium equation applied over the domain of the characteristic volume, $\Theta$. Equation 14 is averaged over $\Theta$ to obtain the macroscopic equilibrium equation defined over the problem domain, $\Omega$. 


\subsection{Eigendeformation based reduced order homogenization model}

The above procedure leads to the standard computational homogenization (also known as $\mathrm{FE}^{2}$ ) method. While accurate, direct implementation of this approach is computationally costly. Instead, we employ the symmetric eigendeformation-based reduced order homogenized method (sEHM) originally proposed in Refs. $[8,11]$. For brevity, a brief overview of the reduced order homogenization is presented below. The microscale displacement field is defined by the heterogeneous morphology of the microstructure, the macroscopic applied strains, and the inelastic strains at the microscale as:

$$
\mathbf{u}^{1}=\mathbf{H}(\mathbf{y}): \overline{\boldsymbol{\epsilon}}(\mathbf{x}, t)+\int_{\Theta} \mathbf{h}(\mathbf{y}, \hat{\mathbf{y}}): \boldsymbol{\mu}(\mathbf{x}, \hat{\mathbf{y}}, t) d \hat{\mathbf{y}}
$$

where $\mathbf{H}$ is the elastic influence function and $\mathbf{h}$ is the phase damage induced influence function obtained from the elastic behavior of the microstructure in the absence and presence of damage, respectively.

Significant computational savings are achieved by precomputing pertinent information about the response of the characteristic volume using a reduced number of degrees of freedom describing the microstructure response. The characteristic volume domain, $\Theta$, is partitioned into $n$ nonoverlapping subdomains, $\theta^{(\gamma)}$, referred to as parts, where $\gamma=1,2, \ldots$ indicates the part number. The partitioning of the reduced model parts are performed such that parts do not intersect both constituents. Phase shape functions, $N^{(\gamma)}$, are defined as piecewise constant functions which form a partition of unity in the characteristic volume (i.e. $N^{(\gamma)}=1$ if $\mathbf{y} \in \theta^{(\gamma)}$ and 0 otherwise). The inelastic strains and scalar damage values are taken to be constant over a given part and the inelastic strains at any point in the partitioned microstructure are given by:

$$
\boldsymbol{\mu}(\mathbf{x}, \mathbf{y}, t)=\sum_{\gamma} N^{(\gamma)}(\mathbf{y}) \boldsymbol{\mu}^{(\gamma)}(\mathbf{x}, t)
$$

Similarly, the piecewise constant damage in the microstructure is approximated as:

$$
\omega(\mathbf{x}, \mathbf{y}, t)=\sum_{\gamma} N^{(\gamma)}(\mathbf{y}) \omega^{(\gamma)}(\mathbf{x}, t)
$$

The parts of the RVE are partitioned so as to group the similar regions of the microstructure corresponding to the macroscopic failure modes. The damage induced inelastic strains, or eigenstrains,

$\boldsymbol{\mu}^{(\gamma)}$, are found by solving a non-linear system of equations defined by the macroscopic strain state, elastic influence functions, phase damage influence functions, phase shape functions, and the internal damage variables, $\omega^{(\gamma)}$, from each part. The macroscopic stress field, $\overline{\boldsymbol{\sigma}}$, is calculated as the homogenized stress over the entire microstructure including the contributions from all parts. The resulting coupled macroscale and microscale boundary value problems are summarized below. 
Macroscale boundary value problem: Given average body force $\overline{\mathbf{b}}$ and boundary conditions $\hat{\mathbf{u}}$ and $\hat{\mathbf{t}}$, find the macroscopic displacement field, $\overline{\mathbf{u}}$, such that:

$$
\begin{gathered}
\nabla_{x} \cdot \overline{\boldsymbol{\sigma}}(\mathbf{x}, t)+\overline{\mathbf{b}}(\mathbf{x})=0 ; \quad \mathbf{x} \in \Omega \\
\overline{\boldsymbol{\epsilon}}=\nabla_{y}^{s} \overline{\mathbf{u}} \\
\overline{\mathbf{u}}=\hat{\mathbf{u}}(\mathbf{x}, t) ; \quad \mathbf{x} \in \Gamma_{u} \\
\overline{\boldsymbol{\sigma}}(\mathbf{x}, t) \cdot \mathbf{n}=\hat{\mathbf{t}}(\mathbf{x}, t) ; \quad \mathbf{x} \in \Gamma_{t}
\end{gathered}
$$

The homogenized macroscale stress is obtained by solving the reduced order microscale boundary value problem.

Microscale boundary value problem: Given macroscale strain state, $\overline{\boldsymbol{\epsilon}}$, and damage state in each part of the microstructure, $\omega^{(\gamma)}$, defined by the evolution equations for part-average damage in each part (provided below); find eigenstrains in each part, $\boldsymbol{\mu}^{(\gamma)}$, such that,

$$
\sum_{\Delta=1}^{n}\left\{\left[1-\omega^{(\Delta)}\right]\left[\hat{\mathbf{A}}^{(\alpha \Delta)}: \overline{\boldsymbol{\epsilon}}+\sum_{\gamma=1}^{n} \hat{\mathbf{B}}^{(\alpha \Delta \gamma)}: \boldsymbol{\mu}^{(\gamma)}\right]\right\}=0 \quad \forall \alpha=1,2, \ldots, n
$$

in which $n$ is the number of parts in $\Theta$, and $\hat{\mathbf{A}}^{(\alpha \Delta)}$ and $\hat{\mathbf{B}}^{(\alpha \Delta \gamma)}$ are coefficient tensors computed from the elastic influence function, phase damage influence function, and elastic properties of the constituents.

The homogenized macroscopic stress is computed as a function of the part-average damage and eigenstrain coefficients as:

$$
\overline{\boldsymbol{\sigma}}=\sum_{\Delta=1}^{n}\left[1-\omega^{(\Delta)}\right]\left[\overline{\mathbf{L}}^{(\Delta)}: \overline{\boldsymbol{\epsilon}}+\sum_{\alpha=1}^{n} \overline{\mathbf{P}}^{(\alpha \Delta)}: \boldsymbol{\mu}^{(\alpha)}\right]
$$

where $\overline{\mathbf{L}}^{(\Delta)}$ and $\overline{\mathbf{P}}^{(\alpha \Delta)}$ are coefficient tensors. The exact expressions of the coefficient tensors and their derivations are provided in Ref. [8] and omitted here for brevity. Since the microstructural topology and elastic constants of the constituents are taken to be deterministic, the coefficient tensors are deterministic quantities as well. The homogenized macroscopic stress in Eq. 23 constitutes the stress update in the solution to the macroscale boundary value problem.

\subsection{Damage Evolution}

The evolution of the reduced order characteristic volume problem requires the constitutive relationship between the part average microstructure field variables. In this regard, a rate-dependent damage evolution model is employed. The accumulation of damage in constituent part $\theta^{(\gamma)}$ is driven 
by the internal state variables of phase damage equivalent strain, $v^{(\gamma)}$, and the monotonically increasing phase damage hardening variable, $r^{(\gamma)}$. The damage potential function $f$ is expressed as:

$$
f\left(v^{(\gamma)}, r^{(\gamma)}\right)=\phi\left(v^{(\gamma)}\right)-\phi\left(r^{(\gamma)}\right)
$$

in which $\phi$ is the damage evolution function given by the power law relationship

$$
\phi^{(\gamma)}(v)=a^{(\gamma)}\left\langle v-v_{0}^{(\gamma)}\right\rangle^{b^{(\gamma)}}
$$

where $a^{(\gamma)}$ and $b^{(\gamma)}$ are material parameters, $v_{0}^{(\gamma)}$ is the threshold strain state below which no damage accumulates in the material, $v$ is $v^{(\gamma)}$ or $r^{(\gamma)}$, and $\langle\cdot\rangle$ indicates Macaulay brackets: $\langle\cdot\rangle=\frac{(\cdot)+\|\cdot\|}{2}$. Phase damage equivalent strain is determined from the average strain in a part, $\boldsymbol{\epsilon}^{(\gamma)}$, by the relationship

$$
v^{(\gamma)}\left(\boldsymbol{\epsilon}^{(\gamma)}\right)=\sqrt{\frac{1}{2}\left(\mathbf{F}^{(\gamma)} \hat{\boldsymbol{\epsilon}}^{(\gamma)}\right)^{\top} \mathbf{L}^{(\gamma)}\left(\mathbf{F}^{(\gamma)} \hat{\boldsymbol{\epsilon}}^{(\gamma)}\right)}
$$

where $\hat{\boldsymbol{\epsilon}}^{(\gamma)}$ is the principal components vector of the part average strain tensor, $\boldsymbol{\epsilon}^{(\gamma)}, \mathbf{L}^{(\gamma)}$ is the tensor of elastic moduli in the principal directions of $\boldsymbol{\epsilon}^{(\gamma)}$ for the constituent material that occupies part $\gamma, \mathbf{F}^{(\gamma)}$ is the tension/compression anisotropy weighting matrix, and the superscript $\mathrm{T}$ denotes the matrix transpose. The weighting matrix allows damage accumulation to depend on whether the strains are in tension or compression, where

$$
\begin{gathered}
\mathbf{F}^{(\gamma)}=\left[\begin{array}{ccc}
h_{1}^{(\gamma)} & 0 & 0 \\
0 & h_{2}^{(\gamma)} & 0 \\
0 & 0 & h_{3}^{(\gamma)}
\end{array}\right] \\
h_{\xi}^{(\gamma)}=\frac{1}{2}+\frac{1}{\pi} \operatorname{atan}\left[c_{1}^{(\gamma)}\left(\hat{\epsilon}_{\xi}^{(\gamma)}-c_{2}^{(\gamma)}\right)\right]
\end{gathered}
$$

in which $c_{1}^{(\gamma)}$ and $c_{2}^{(\gamma)}$ are material parameters. The evolution of the phase damage hardening variable and microscopic damage are expressed as:

$$
\begin{gathered}
\dot{r}^{(\gamma)}=\dot{\lambda} \\
\dot{\omega}^{(\gamma)}=\dot{\lambda} \frac{\partial \phi}{\partial v^{(\gamma)}} .
\end{gathered}
$$

For the rate dependent damage law, the consistency parameter $\dot{\lambda}$ is given as:

$$
\dot{\lambda}=\frac{1}{q^{(\gamma)}}\left\langle f\left(v^{(\gamma)}, r^{(\gamma)}\right)\right\rangle^{p^{(\gamma)}}
$$

where $p^{(\gamma)}$ and $q^{(\gamma)}$ are material parameters that govern rate-dependency [35]. 
The above rate-dependent damage evolution model contains seven parameters describing the failure evolution in a part. Every part that lays within the subdomain of the same constituent is associated with an identical parameter set. For instance, given $\boldsymbol{\psi}^{(m)}=\left\{a^{(m)}, b^{(m)}, v_{0}^{(m)}, p^{(m)}, q^{(m)}, c_{1}^{(m)}, c_{2}^{(m)}\right\}$ is the set of random parameters defining failure in the matrix and the set of random fiber failure parameters, $\boldsymbol{\psi}^{(f)}$, is defined similarly, then:

$$
\begin{cases}\boldsymbol{\psi}^{(\gamma)}=\boldsymbol{\psi}^{(m)} & \text { if } \theta^{(\gamma)} \in \theta^{(m)} \\ \boldsymbol{\psi}^{(\gamma)}=\boldsymbol{\psi}^{(f)} & \text { if } \theta^{(\gamma)} \in \theta^{(f)}\end{cases}
$$

where $\theta^{(m)}$ and $\theta^{(f)}$ denote the subdomains of the characteristic volume occupied by the matrix and fiber, respectively. The set of random failure parameters of the proposed model is therefore:

$$
\boldsymbol{\psi}=\left\{\boldsymbol{\psi}^{(m)}, \boldsymbol{\psi}^{(f)}\right\}
$$

\section{Calibration of Composite Multiscale Model under Un- certainty}

This section describes the Bayesian statistical approach employed in the calibration of the multiscale model in the presence of uncertainty. Bayesian methods are introduced to calibrate random parameter distributions from sparse and uncertain data. Markov chain Monte Carlo simulation (MCMC) is used to evaluate the probability distributions of parameters given their predicted simulation outputs. Since MCMC requires a large number of forward simulations, Gaussian process (GP) surrogate models are employed to approximate the response surfaces of the multiscale model for computational efficiency.

\subsection{Bayesian Calibration}

In this study, the Bayesian statistical approach is employed to calibrate the probabilistic distributions of the constituent failure parameters and quantify their uncertainty. A critical observation is that the calibration process itself is multiscale. Calibration data is often measured and meaningful at the laminate level, whereas the parameters to be calibrated are characterized at the scale of the characteristic volume.

Consider an arbitrary quantity of interest, $\phi_{\text {lam }}$, (e.g., stress to failure) that is measurable at the scale of the laminate. Assume the macroscale quantity can be described as a function, $G$, of the microscale material parameters, $\psi$ :

$$
\phi_{\text {lam }}=G(\boldsymbol{\psi}) \quad \boldsymbol{\psi} \in \mathbf{S} \subset \mathbb{R}^{n_{p}}
$$


where $n_{p}$ is the number of constituent parameters, and $\mathbf{S}$ the range of parameter values defined by physical constraints. In this paper, $G(\boldsymbol{\psi})$ is the response surface defined by the multiscale model. Under the deterministic approach, $\phi_{\text {lam }}$ is a scalar value and the goal of the calibration process is to find a single vector of constituent parameters which produce the desired output. This is analogous to solving the inverse problem, $\boldsymbol{\psi}=G^{-1}\left(\phi_{\text {lam }}\right)$. For multiscale analyses with non-linear material models, $G$ is not easily invertible.

In a probabilistic setting, the desire is not only to find the parameters resulting in a single value of $\phi_{\text {lam }}$, but the distribution of parameters, $f_{\psi}$, which lead to the desired random distribution in the quantity of interest, $f_{\phi_{l a m}}$. The probabilistic constituent material parameter calibration strategy employed herein is rooted in Bayes' Theorem. Consider two distinct random events $A$ and $B$. The probability of simultaneously observing $A$ and $B$, denoted as $P(A B)$, is expressed using the conditional probabilities as:

$$
P(A B)=P(A \mid B) P(B)=P(B \mid A) P(A)
$$

where, $0 \leq P(A) \leq 1$ denotes the probability of observing event $A$, and $P(A \mid B)$ the conditional probability of observing $A$ given $B$ has been observed. Rearranging the equality yields Bayes' Theorem:

$$
P(A \mid B)=\frac{P(B \mid A) P(A)}{P(B)} ; \quad P(B)>0
$$

Considering the collectively exhaustive and mutually exclusive events $A_{i}(i \in \mathcal{I}:=\{1,2 \ldots, m\})$ where $\sum_{i} P\left(A_{i}\right)=1$ and $P\left(A_{j}\right) \geq 0 \forall j \in \mathcal{I}$, and using the theorem of total probability, Bayes' Theorem is expressed as:

$$
P\left(A_{i} \mid B\right)=\frac{P\left(B \mid A_{i}\right) P\left(A_{i}\right)}{\sum_{j} P\left(B \mid A_{j}\right) P\left(A_{j}\right)}
$$

Equation 37 is interpreted as a probability update procedure. Let $B$ be an event, such as an observation from an experiment, that has some dependence on $A_{i}$, such that $P\left(B \mid A_{i}\right)$ is well defined, then $P\left(A_{i}\right)$ and $P\left(A_{i} \mid B\right)$ are interpreted as the probability distributions of $A_{i}$ prior to and posterior to the occurrence of event $B$, respectively. In the context of the material parameter updating, $A_{i}$ are considered to be continuous rather than discrete events. Renaming the variables and considering continuous events, the parameter update function is expressed as:

$$
f\left(\boldsymbol{\psi} \mid y_{o b s}\right)=\frac{f\left(y_{o b s} \mid \boldsymbol{\psi}\right) f(\boldsymbol{\psi})}{\int_{\psi} f\left(y_{o b s} \mid \mathbf{x}\right) f(\mathbf{x}) d \mathbf{x}}
$$

in which $y_{o b s}$ denotes observed experimental data, $f\left(\boldsymbol{\psi} \mid y_{o b s}\right)$ is the posterior probability of the parameters $\boldsymbol{\psi}$ given the observed experimental data, $f\left(y_{o b s} \mid \boldsymbol{\psi}\right)$ the probability of observing $y_{o b s}$ given parameters $\boldsymbol{\psi}$, and $f(\boldsymbol{\psi})$ the prior probability of $\boldsymbol{\psi}$. The conditional probability, $f\left(y_{o b s} \mid \boldsymbol{\psi}\right)$, is the "likelihood" of parameters $\boldsymbol{\psi}$ producing $y_{\text {obs }}$, and is denoted as $\mathcal{L}(\boldsymbol{\psi})$. For simplicity, prior 
and posterior probabilities of $\boldsymbol{\psi}$ are denoted as $f^{\prime}(\boldsymbol{\psi})$ and $f^{\prime \prime}(\boldsymbol{\psi})$, respectively. The denominator of the right hand side in Eq. (38) is a normalizing constant such that the area under the curve of $f^{\prime \prime}(\boldsymbol{\psi})$ is equal to 1 . As will be seen below, the actual value of $f^{\prime \prime}(\boldsymbol{\psi})$ is not important in the Bayesian updating procedure, only the ratio between the probability of different parameter points, and therefore the expensive integration required to evaluate the denominator does not need to be performed. The parameter update is then expressed in the alternative form:

$$
f^{\prime \prime}(\boldsymbol{\psi}) \propto \mathcal{L}(\boldsymbol{\psi}) f^{\prime}(\boldsymbol{\psi})=\bar{f}(\boldsymbol{\psi})
$$

where $\bar{f}(\boldsymbol{\psi})$ is the unnormalized kernel of the posterior probability distribution for $\boldsymbol{\psi}$.

When the observations come from a suite of similar or identical experiments, the observation, $y_{o b s}$, is a distribution characterized by the probability density function, $f_{\text {obs }}$. The observation distribution can be expressed using either a parameterized distribution (i.e normal, log-normal, Weibull, etc.) or in a non-parametric form, such as the Kernel Spectral (KS) function [27, 25].

Consider a set of experimental observations, $\mathbf{y}_{o b s}=\left\{y_{o b s}^{1}, y_{o b s}^{2}, \ldots, y_{o b s}^{p}\right\}$, the probability density using the KS approximation is expressed as:

$$
f_{o b s}(y) \simeq \frac{1}{p h} \sum_{i=1}^{p} K\left(\frac{y-y_{o b s}^{i}}{h}\right)
$$

where $p$ is the total number of observations, $h$ the kernel bandwidth, and $K$ the symmetric kernel. In the form of the standard normal density function,

$$
K(x)=\frac{1}{\sqrt{2 \pi}} \exp \left(-\frac{1}{2} x^{2}\right) .
$$

From Ref. [34], the optimal bandwidth for a standard normal kernel is estimated as:

$$
h=\left(\frac{4 \sigma_{o b s}^{5}}{3 p}\right)^{\frac{1}{5}}
$$

where $\sigma_{o b s}$ is the standard deviation of the experimental observations.

When the experimental observation is a scalar (e.g., strength), using KS density estimation and recalling that the model prediction of the observed quantity, $\phi_{\text {lam }}$, is expressed in terms of the constituent parameters as: $\phi_{\text {lam }}=G(\boldsymbol{\psi})$, the likelihood function is defined as:

$$
\mathcal{L}(\boldsymbol{\psi})=\frac{1}{p h} \frac{1}{\sqrt{2 \pi}} \sum_{i=1}^{p} \exp \left(-\frac{1}{2}\left[\frac{G(\boldsymbol{\psi})-y_{\text {obs }}^{i}}{h}\right]^{2}\right) .
$$




\subsection{Accelerated Sampling using Markov Chain Monte Carlo Simulation}

Markov chain Monte Carlo simulation is used to produce a chain of failure parameter values from Monte Carlo simulation whose probability density estimates the posterior distribution of those parameters. In general, a Markov chain is a memoryless random process where a given state depends on the previous state only. In the context of parameter updating, the states in the Markov chain are the parameter values, and each new state is determined by comparing the likelihood estimate of the previous state and a trial state. Given a parameter state in the chain $\boldsymbol{\psi}_{k}$, a trial parameter state $\boldsymbol{\psi}^{*}$ is sampled by taking a random walk from $\boldsymbol{\psi}_{k}$. Considering the prior probability times likelihood of the two parameter sets, $\bar{f}\left(\boldsymbol{\psi}_{k}\right)=\mathcal{L}\left(\boldsymbol{\psi}_{k}\right) f^{\prime}\left(\boldsymbol{\psi}_{k}\right)$ and $\bar{f}\left(\boldsymbol{\psi}^{*}\right)=\mathcal{L}\left(\boldsymbol{\psi}^{*}\right) f^{\prime}\left(\boldsymbol{\psi}^{*}\right)$. The trial state, $\boldsymbol{\psi}^{*}$, is accepted with probability of $a=\min \left(1, \bar{f}\left(\boldsymbol{\psi}^{*}\right) / \bar{f}\left(\boldsymbol{\psi}_{k}\right)\right)$. If $\boldsymbol{\psi}^{*}$ is rejected, $\boldsymbol{\psi}_{k}$ is repeated in the chain and a new $\boldsymbol{\psi}^{*}$ is sampled and tested.

The completion criteria for MCMC is the convergence of the chain. Chain convergence is achieved when additional samples do not effect the generated distributions. The posterior density distributions of each of the parameters can then be evaluated by fitting the chain to a parametric distribution, or employing KS density for a non-parametric PDF. Additionally, the covariance of the parameters in the chain is calculated as:

$$
\sigma_{c o v}\left(\boldsymbol{\psi}^{i}, \boldsymbol{\psi}^{j}\right)=\frac{1}{N_{m c m c}-1} \sum_{k=1}^{N_{m c m c}}\left(\boldsymbol{\psi}_{k}^{i}-\overline{\boldsymbol{\psi}}^{i}\right)\left(\boldsymbol{\psi}_{k}^{j}-\overline{\boldsymbol{\psi}}^{j}\right)
$$

where $N_{m c m c}$ is the number of MCMC steps and $\bar{\psi}^{i}$ is the sample mean of $\boldsymbol{\psi}^{i}$, which is the chain of parameters generated for the $i^{\text {th }}$ component of $\boldsymbol{\psi}$. The covariance is important in sampling correlated random parameters from the generated distributions.

\subsection{Surrogate models using Gaussian processes}

MCMC requires hundreds of thousands of samples evaluated in serial to generate converged parameter chains. The time required to perform this procedure using the nonlinear multiscale simulations is prohibitively large. To achieve computational efficiency, a surrogate model is trained to approximate the response surface which is otherwise generated by full multiscale simulations. In this manuscript, Gaussian process (GP) modeling is employed because of its ability to capture nonlinear surfaces and quantify the uncertainty in the approximation of the response surface [18, 26, 31].

The GP model is a variant of a radial basis function built on Gaussian kernels. The GP model is trained by a series of simulations performed using the multiscale model. Consider a set of $n_{t}$ input training parameter sets, $\boldsymbol{\psi}_{t}=\left\{\boldsymbol{\psi}_{t_{1}}, \ldots, \boldsymbol{\psi}_{t_{n t}}\right\}$ such that $\boldsymbol{\psi}_{t_{i}} \in \mathbf{S}$. In this work, Latin hypercube sampling is employed to obtain parameter sets spread within $\mathbf{S}$. The multiscale model is exercised to compute the corresponding set of output values, $\mathbf{y}_{t}$, from parameters $\boldsymbol{\psi}_{t}$. For a given input prediction point, $\boldsymbol{\psi}_{p}$, the output of the GP, $y_{p}$, is computed as a Gaussian distribution conditioned 
upon the prediction point, training points, training point outputs, and the hyperparameters of the GP, $\Psi$ :

$$
p\left(y_{p} \mid \boldsymbol{\psi}_{p}, \boldsymbol{\psi}_{t}, \mathbf{y}_{t} ; \boldsymbol{\Psi}\right) \sim N(m, S)
$$

where $m$ and $S$ are the prediction mean and variance and $N$ denotes a Gaussian distribution.

The covariance, $k_{i j}$, between two points, $\boldsymbol{\psi}_{i}$ and $\boldsymbol{\psi}_{j}$, in the input space is defined as

$$
k_{i j}=k\left(\boldsymbol{\psi}_{i}, \boldsymbol{\psi}_{j}\right)=\theta_{1} \exp \left\{-\frac{1}{2} \sum_{d=1}^{n_{p}} \frac{\left(\psi_{i_{d}}-\psi_{j_{d}}\right)^{2}}{\lambda_{d}^{2}}\right\}
$$

in which $\theta_{1}$ is a scaling factor and $\lambda_{d}$ the length scale parameter in the $d^{\text {th }}$ input dimension. Covariance matrices can then be constructed between two sets of input points. A covariance matrix is defined for any two sets of parameters, $\boldsymbol{\psi}_{a}=\left\{\boldsymbol{\psi}_{a_{1}}, \ldots, \boldsymbol{\psi}_{a_{n a}}\right\}$ and $\boldsymbol{\psi}_{b}=\left\{\boldsymbol{\psi}_{b_{1}}, \ldots, \boldsymbol{\psi}_{b_{n b}}\right\}$ such that

$$
\mathbf{K}_{A B_{i j}}=k\left(\boldsymbol{\psi}_{a_{i}}, \boldsymbol{\psi}_{b_{i}}\right)
$$

As such, the covariance matrices between the training points, $\boldsymbol{\psi}_{t}$, and prediction points $\boldsymbol{\psi}_{p}$ are constructed and the prediction mean and variance are defined as

$$
\begin{gathered}
m=\mathbf{K}_{P T}\left(\mathbf{K}_{T T}+\sigma_{n}^{2} I\right)^{-1} \mathbf{y}_{t} \\
S=\mathbf{K}_{P P}-\mathbf{K}_{P T}\left(\mathbf{K}_{T T}+\sigma_{n}^{2} I\right)^{-1} \mathbf{K}_{T P}
\end{gathered}
$$

where $\sigma_{n}^{2}$ is the noise variance of the training points and $I$ indicates the identity matrix whose size matches $\mathbf{K}_{T T}$. For training point predictions from computer models, $\sigma_{n}^{2}$ is taken as 0 , as the computer prediction is taken to be a noiseless process (i.e. the computer output is taken to be always the same for the same input).

\section{Calibration and Validation}

Random failure parameters for the fiber and matrix constituents of IM7/977-3 composites were probabilistically calibrated using experimental data from $0^{\circ}$ and $90^{\circ}$ unidirectional specimens at varying load rates. The proposed multiscale model with random failure parameters is employed to predict the failure strength for a quasi-isotropic IM7/977-3 laminated composite with an open hole under tension at a fast and slow loading rate to demonstrate the rate-dependence and uncertainty quantification of the model. These results are compared to experiments. The effect of spatial variability on failure parameters in predictions of the quasi-isotropic laminate failure strength is also investigated. 


\section{$4.1 \quad$ Experiments}

A suite of experiments was conducted to calibrate the material parameters and validate the proposed computational model for rate dependent damage accumulation in carbon fiber reinforced epoxy composite laminates. IM7/977-3 composite specimens with three separate layups of unidirectional laminae were tested: (a) $0^{\circ}$ specimens consisting of eight unidirectional plies with fibers oriented parallel to the coupon length; (b) $90^{\circ}$ specimens consisting of sixteen unidirectional plies with the fibers oriented perpendicular to the coupon length; and (c) open-hole quasi-isotropic specimens with a layup of $[+45,0,-45,90]_{2 s}$ and hole diameter of $6 \mathrm{~mm}$. Specimen configurations are summarized in Table 1. The mean fiber volume fraction of the specimens was determined to be $65 \%$ based on acid digestion testing. Monotonic tension experiments were conducted on the $0^{\circ}$ specimens according

Table 1: IM7/977-3 Nominal Specimen Dimensions.

\begin{tabular}{cccccc}
\hline $\begin{array}{c}\text { Fiber } \\
\text { Orientation }\end{array}$ & $\begin{array}{c}\text { Number } \\
\text { Of Plies }\end{array}$ & $\begin{array}{c}\text { Length } \\
(\mathrm{mm})\end{array}$ & $\begin{array}{c}\text { Width } \\
(\mathrm{mm})\end{array}$ & $\begin{array}{c}\text { Thickness } \\
(\mathrm{mm})\end{array}$ & $\begin{array}{c}\text { \# specimens } \\
\text { tested }\end{array}$ \\
\hline $0^{\circ}$ & 8 & 250 & 13 & 1 & 12 \\
$90^{\circ}$ & 16 & 100 & 13 & 2 & 25 \\
{$[+45,0,-45,90]_{2 s}$} & 16 & 250 & 38 & 2 & 6 \\
\hline
\end{tabular}

to ASTM Standard D3039 [1] at a quasi-static displacement rate of $1.0 \mathrm{~mm} / \mathrm{min}$. Strain in the $0^{\circ}$ specimens was measured using a centrally located extensometer. Applied load was measured directly from the testing machine and stress was calculated over the gross cross section of the specimen. Three point bend tests were performed on the $90^{\circ}$ specimens with a span length of $44.7 \mathrm{~mm}$ at midpoint displacement rates of $0.1 \mathrm{~mm} / \mathrm{min}, 1.0 \mathrm{~mm} / \mathrm{min}$, and $100 \mathrm{~mm} / \mathrm{min}$ according to ASTM Standard D790 [3]. The midpoint displacement and applied load were measured directly from the testing machine. Stress and strain at the bottom of the beam at midspan were calculated from elastic beam theory. The quasi-isotropic specimens were tested according to ASTM Standard D5766 [2] at displacement rates of $1.0 \mathrm{~mm} / \mathrm{min}$ and $100 \mathrm{~mm} / \mathrm{min}$. Strain was measured using a 1-inch long extensometer centered on the hole. Applied load was measured from the testing machine and stress was computed over the gross cross-sectional area of the specimen. All tests were performed on an MTS universal testing machine.

\subsection{Model Calibration}

The geometry and sEHM parts used in the reduced order modeling of material failure are illustrated in Fig. 3. 1206 tetrahedral elements were used in the microscale finite element mesh from which the influence functions and the coefficient tensors of the reduced order model were computed. A four part ROM partition was employed, with part 1 consisting of the carbon fiber and parts 2-4 
consisting of the epoxy resin. The partitioning was defined such that the three major failure modes of the composite are represented, namely fiber failure, transverse matrix cracking, and delamination. The elastic parameters for the constituent materials are shown in Table 2, where $f$ denotes the fiber and $m$ the matrix [10]. The IM7 fiber was treated as a transversely isotropic material. The 977-3 resin was modeled as isotropic. Damage was taken to accumulate only when the constituent phase is in tension.

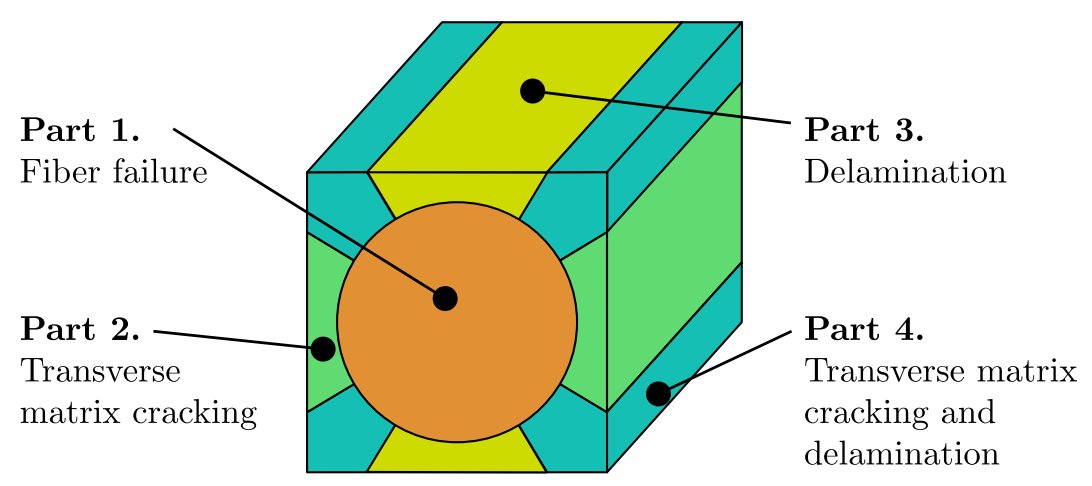

Figure 3: Partitioned RVE.

Table 2: Elastic parameters of fiber and matrix.

\begin{tabular}{ccccccc}
\hline$E^{(m)}[\mathrm{GPa}]$ & $E_{1}^{(f)}[\mathrm{GPa}]$ & $E_{2}^{(f)}[\mathrm{GPa}]$ & $G_{12}^{(f)}[\mathrm{GPa}]$ & $v^{(m)}$ & $v_{12}^{(f)}$ & $v_{23}^{(f)}$ \\
\hline 3.55 & 263.0 & 13.0 & 27.5 & 0.35 & 0.32 & 0.20 \\
\hline
\end{tabular}

A total of 14 material parameters ( 7 for the matrix and 7 for the fiber) define the rate dependent failure behavior of the composite constituents, as outlined in Table 3. For both constituents, the damage thresholds $\left(v_{0}^{(m)}\right.$ and $\left.v_{0}^{(f)}\right)$ were taken to vanish, implying that damage accumulation occurs from the onset of loading. The tension-compression anisotropy parameters $\left(c_{1}^{(m)}, c_{2}^{(m)}, c_{1}^{(f)}\right.$, and $\left.c_{2}^{(f)}\right)$ were set such that damage accumulates only under tensile loading (compression loading is not considered in this study). Because carbon fibers do not exhibit significant rate dependence, $p^{(f)}$ and $q^{(f)}$ were set to 100 and 1 , respectively, which mimics the rate independent limit. The remaining six parameters were calibrated using the proposed Bayesian approach. The results of the $0^{\circ}$ monotonic tension tests were employed to calibrate the failure behavior of the fiber (i.e. $a^{(f)}$ and $b^{(f)}$ ) since fiber failure dominates the failure response in these experiments. The three point bending test results were used to calibrate the matrix failure parameters (i.e. $a^{(m)}, b^{(m)}, p^{(m)}$, and $q^{(m)}$ ). The quasi-isotropic specimens were used to validate the response predictions.

The stress-strain curves from the twelve $0^{\circ}$ monotonic tension tests are shown in Fig. 4. The experimental longitudinal mean failure stress, $X_{1}$, and failure strain, $\epsilon_{1}$, were $2785 \mathrm{MPa}$ and $1.64 \%$, with standard deviations of $297.2 \mathrm{MPa}$ and $0.185 \%$, respectively. The range of failure stress was 
Table 3: Material properties describing damage evolution.

\begin{tabular}{ccc}
\hline Property & Description & Equation \\
\hline$a^{(\gamma)}$ & Governs magnitude of failure & Eq. $(25)$ \\
$b^{(\gamma)}$ & Governs ductility of failure & Eq. $(25)$ \\
$p^{(\gamma)}, q^{(\gamma)}$ & Control rate-dependence & Eq. $(31)$ \\
$c_{1}^{(\gamma)}, c_{2}^{(\gamma)}$ & Control compression/tension anisotropy & Eq. $(28)$ \\
$v_{0}^{(\gamma)}$ & Threshold value of $v^{(\gamma)}$ below which no damage occurs & Eq. $(25)$ \\
\hline
\end{tabular}

$[2435,3300] \mathrm{MPa}$, with failure strain in the range of $[1.26 \%, 1.87 \%]$. All of the $0^{\circ}$ tension tests failed under sudden and catastrophic fiber failure, as seen in Fig. 5.

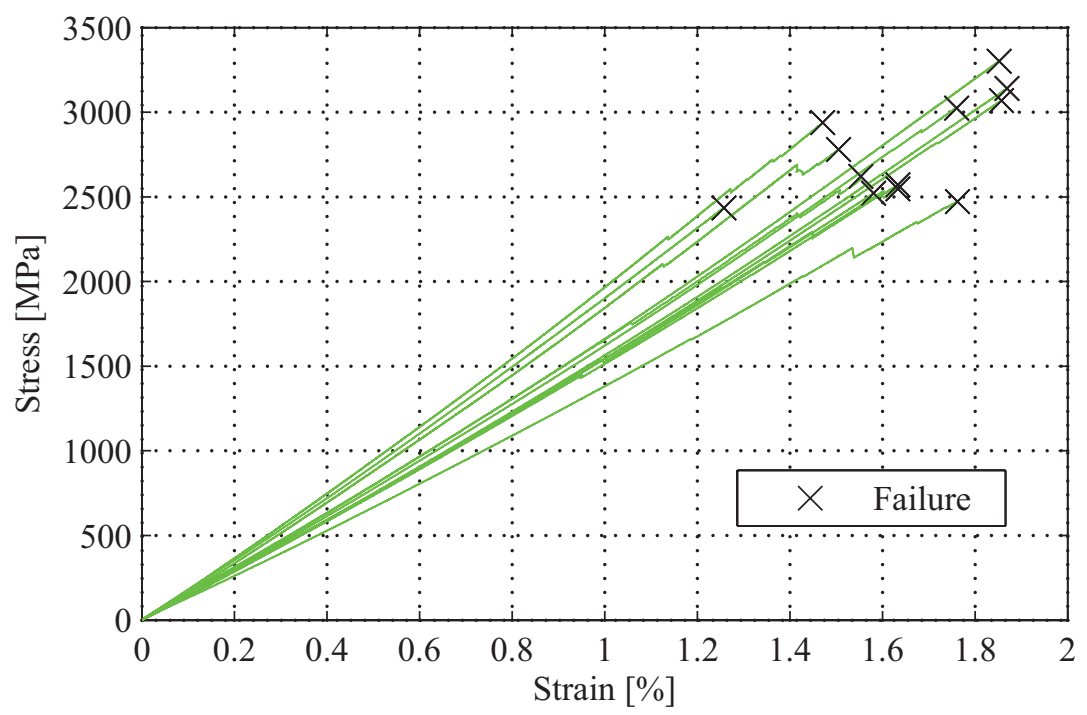

Figure 4: Stress-strain curves for $0^{\circ}$ tension experiments.

The quantity of interest, $\phi_{\text {lam }}$, employed in the calibration procedure was the stress to failure. The 12 failure stress values obtained from the $0^{\circ}$ experiments were used to formulate the likelihood function (Eq. 43) in the MCMC calibration of the fiber damage evolution properties. Fifty multiscale finite element simulations were evaluated using sEHM and the rate-dependent damage evolution law to train the GP surrogate model of the $0^{\circ}$ tension test. The parameters for these simulations were selected using Latin hypercube sampling. One million MCMC steps were performed using the GP model and the resulting chain was used to quantify the distributions of $a^{(f)}$ and $b^{(f)}$. From sampled MCMC chains, probability density functions (PDFs) were computed using kernel spectral density estimation. The covariance matrix $C$ of the parameters was calculated using Eq. (44).

Since the calibration of the model was performed based on the GP model and the GP model is an approximation to the response surface generated by the actual multiscale model, it is necessary to "verify" the calibration procedure directly with the multiscale model predictions. Verification 


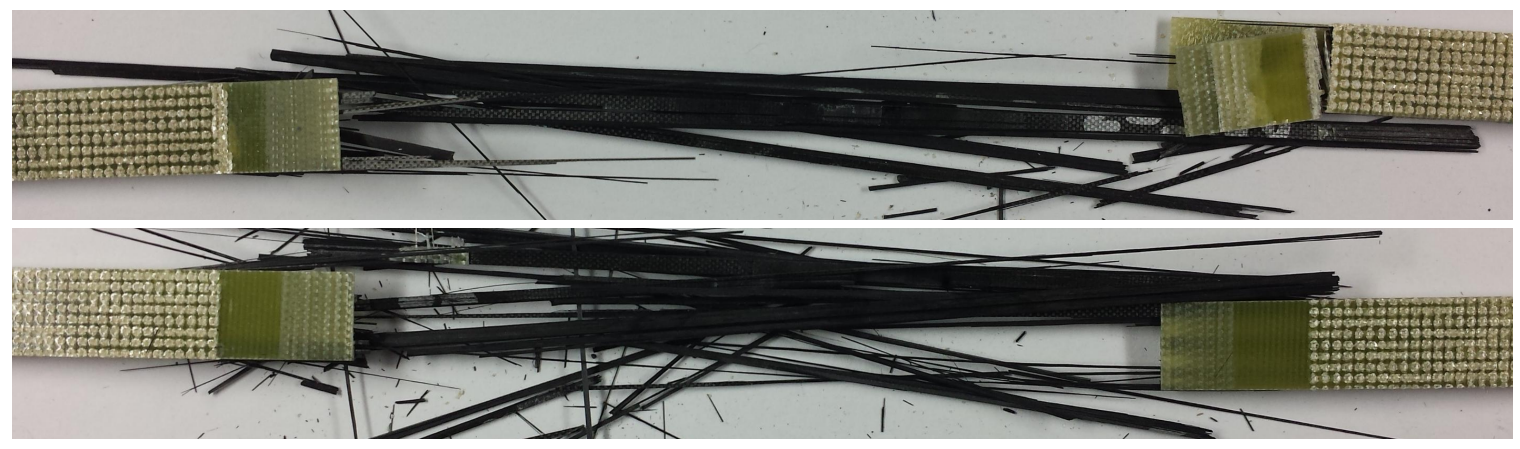

Figure 5: Images of failure from $0^{\circ}$ monotonic tension experiments.

refers to the comparison of the experimental data with full multiscale model simulations performed by drawing samples from the calibrated parameter distributions; i.e., that the calibration process achieved its intended result. The verification of the fiber parameter calibration was conducted by drawing ten thousand samples. The PDF of the verification result is plotted in Fig. 6 against the KS density distribution of experimental results. The longitudinal failure stress and strain properties of the experiments and the calibrated model are summarized in Table 4. The calibrated distributions show good agreement in terms of the statistical mean and coefficient of variation for both stress and strain to failure. We note that the strain to failure data was not used in the generation of the likelihood function in the calibration process, but the resulting verification analysis shows good accuracy to this value.

Table 4: Experimental and calibrated failure properties of unidirectional $0^{\circ}$ specimens.

\begin{tabular}{|c|c|c|c|c|c|}
\hline \multirow[b]{2}{*}{ Property } & & \multicolumn{2}{|c|}{ Experiments } & \multicolumn{2}{|c|}{ Calibration } \\
\hline & & Mean & $\mathrm{CoV}$ & Mean & $\mathrm{CoV}$ \\
\hline Longitudinal failure stress & $X_{1}[\mathrm{MPa}]$ & 2785 & 0.107 & 2752 & 0.124 \\
\hline Longitudinal failure strain & $\epsilon_{1}[\%]$ & 1.64 & 0.113 & 1.74 & 0.125 \\
\hline
\end{tabular}

The mean failure stress from the experiments and simulations are $2785 \mathrm{MPa}$ and $2752 \mathrm{MPa}$, respectively, a difference of $1.2 \%$. The standard deviation of the simulations, $340 \mathrm{MPa}$, is slightly higher than from the experiments, $297 \mathrm{MPa}$. The simulated results show a more exaggerated peak in probability near the cluster of experimental results around $2500 \mathrm{MPa}$. However, the tails of the PDFs of the failure stress for experiments and calibrated model (i.e. Fig. 6(b)) are in good agreement, indicating that the simulations capture the extreme values reasonably well. The mean failure strain from experiments is $1.64 \%$ while the simulations predict a mean of $1.74 \%$. This discrepancy is due to fact that the damage evolution model was calibrated to match the failure stress only, as expressed above.

The failure parameters of the matrix were calibrated using the same procedure. The stressstrain curves of the unidirectional $90^{\circ}$ specimens subjected to three point bending at three separate 


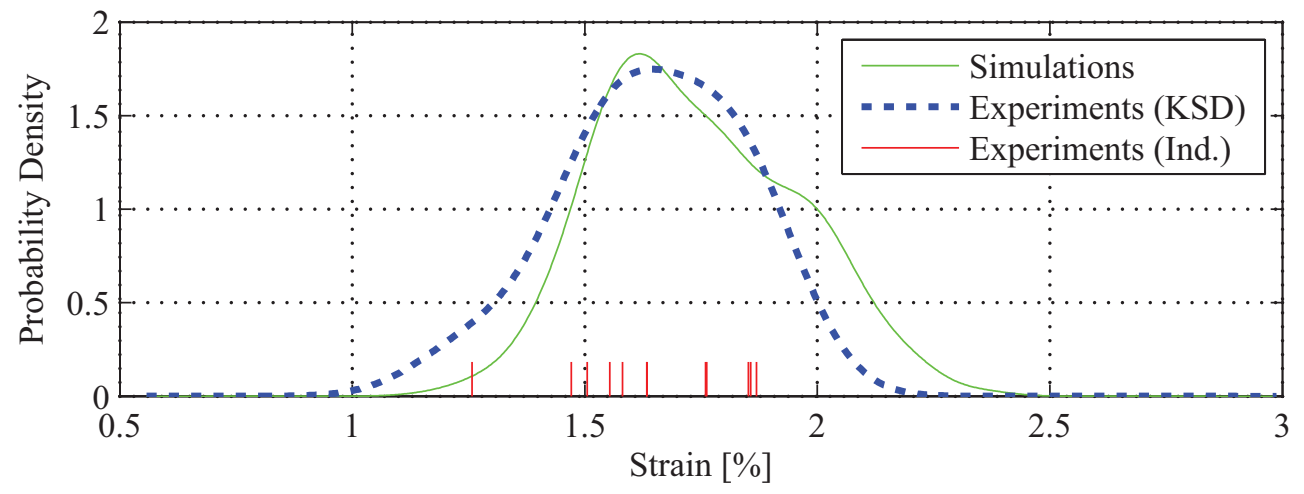

(a)

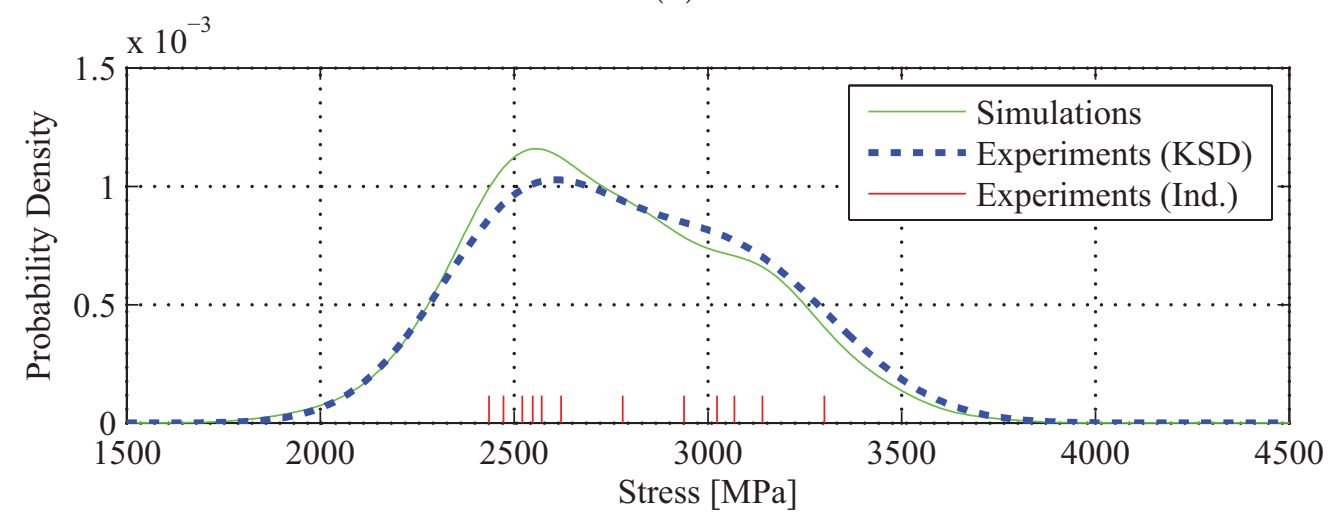

(b)

Figure 6: Experimentally observed and simulated PDFs from $0^{\circ}$ tension specimens: (a) failure strain, (b) failure stress. Tick marks indicate individual experimental values.

loading rates are shown in Fig. 7. The loading rate has a noticeable effect on the failure stress and strain to failure as further illustrated in Fig. 8. The mean transverse failure stress, $X_{2}$, of the specimens increases from 102.3 $\mathrm{MPa}$ at $0.1 \mathrm{~mm} / \mathrm{min}$ to $118.3 \mathrm{MPa}$ at $100 \mathrm{~mm} / \mathrm{min}$ loading rates. Similarly, the mean transverse failure strain, $\epsilon_{2}$, increases from $1.27 \%$ at $0.1 \mathrm{~mm} / \mathrm{min}$ to $1.38 \%$ at $100 \mathrm{~mm} / \mathrm{min}$. The failure stress from all tests is in the range of [82 MPa,135 MPa] and the range of failure strains is $[1.14 \%, 1.61 \%]$. The mode of failure for all of the three point bending experiments was a single smooth matrix crack through the specimen at the midspan, under the loading point. The failure mode was consistent over all of the replicates at each of the loading rates.

The Bayesian calibration procedure was repeated to evaluate the distributions for the matrix failure parameters: $a^{(m)}, b^{(m)}, p^{(m)}$, and $q^{(m)}$. One hundred multiscale simulations were conducted using sampled training points to generate predictions with failure stresses within the range of 50 to $200 \mathrm{MPa}$ and train the GP model. The failure behavior of the composite transverse to the fiber direction was idealized by modeling the critical region of the three point bending specimen at the bottom of the coupon at midspan. One million MCMC steps were performed using the GP model 

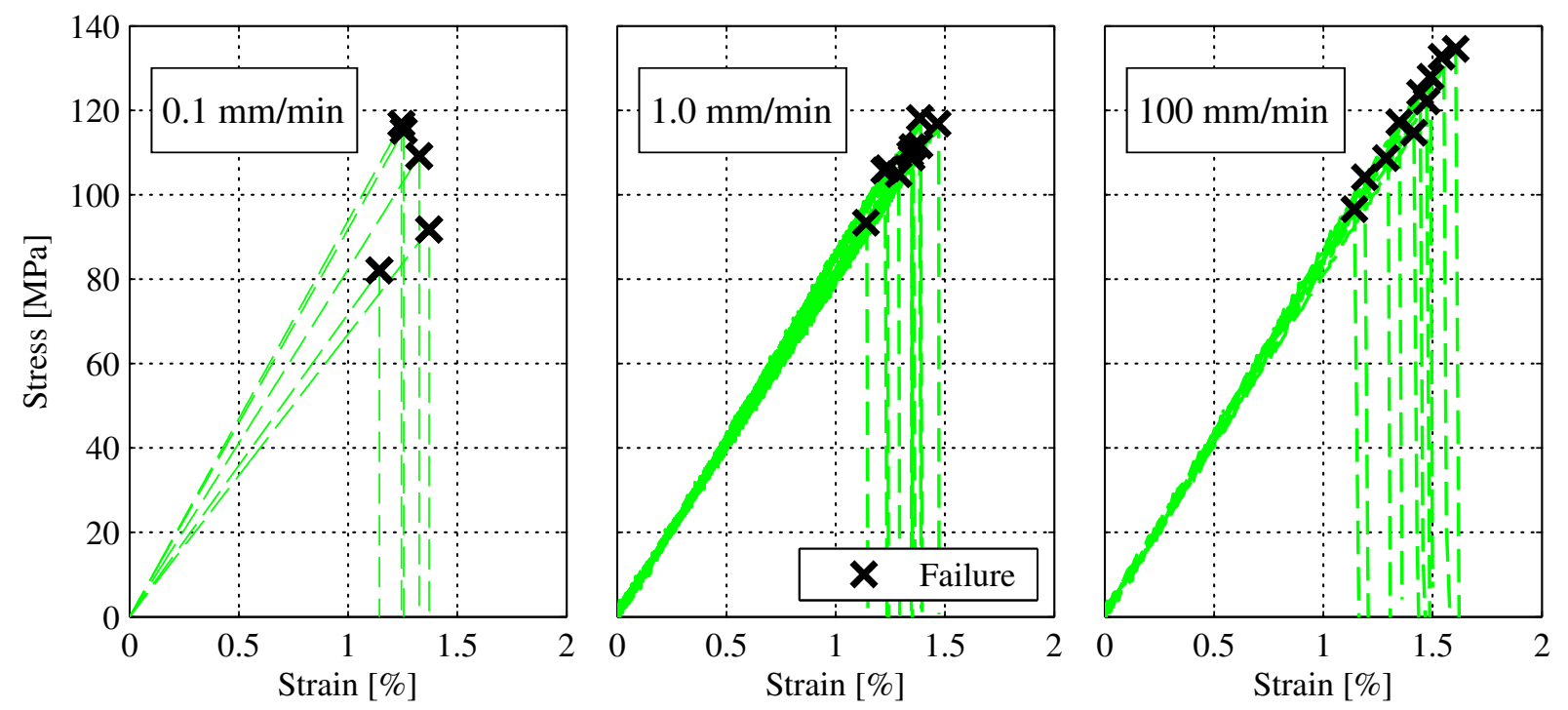

Figure 7: Stress-strain curves for three point bend experiments with $90^{\circ}$ specimens for $0.1 \mathrm{~mm} / \mathrm{min}, 1 \mathrm{~mm} / \mathrm{min}$, and $100 \mathrm{~mm} / \mathrm{min}$ displacement rates.

to generate the distributions of the failure parameters. In order to verify the calibration, random samples were drawn from the KS density distributions and ten thousand simulations were performed using the multiscale model.

Figure 9 compares PDFs of failure stress and failure strain of the three point bend specimens at three loading rates from the multiscale model and the experimental data. The transverse failure stress and strain properties of the experiments and the calibrated model at each displacement rate are summarized in Table 5. The mean failure stresses in the simulations are 104.5, 108.7 and 117.9 MPa for the $0.1 \mathrm{~mm} / \mathrm{min}, 1 \mathrm{~mm} / \mathrm{min}$, and $100 \mathrm{~mm} / \mathrm{min}$ displacement rates, respectively, compared with the experimental means of 102.3, 108.7, and 118.0 MPa. The average stress predictions are in good agreement with the average stress with percent errors of $2.2 \%, 0.0 \%$, and $0.1 \%$ for the $0.1 \mathrm{~mm} / \mathrm{min}, 1 \mathrm{~mm} / \mathrm{min}$, and $100 \mathrm{~mm} / \mathrm{min}$ displacement rates, respectively. While ten experiments were used for the faster rate three point bend tests $(1 \mathrm{~mm} / \mathrm{min}$ and $100 \mathrm{~mm} / \mathrm{min})$, only five were available for the $0.1 \mathrm{~mm} / \mathrm{min}$ rate, which partially explains the slightly higher discrepancy at the slowest rate. The mean failure strain for the slow, medium, and fast rate experiments respectively are $1.27,1.32$, and $1.38 \%$ compared to the mean from simulations of $1.22,1.27$, and $1.38 \%$. The mean failure strain does not correspond to the peak of the PDFs shown in Fig. 7, which is the mode of the PDF and different than the mean for non-Gaussian distributions. The slight discrepancy in the failure strains is attributed to the fact that the likelihood function in the calibration process is computed based on the failure stress and not failure strain.

The standard deviations of failure stress from the experiments are 7.65, 6.97, and 12.44 MPa for the $0.1 \mathrm{~mm} / \mathrm{min}, 1 \mathrm{~mm} / \mathrm{min}$, and $100 \mathrm{~mm} / \mathrm{min}$ displacement rates, respectively. In the calibrated 


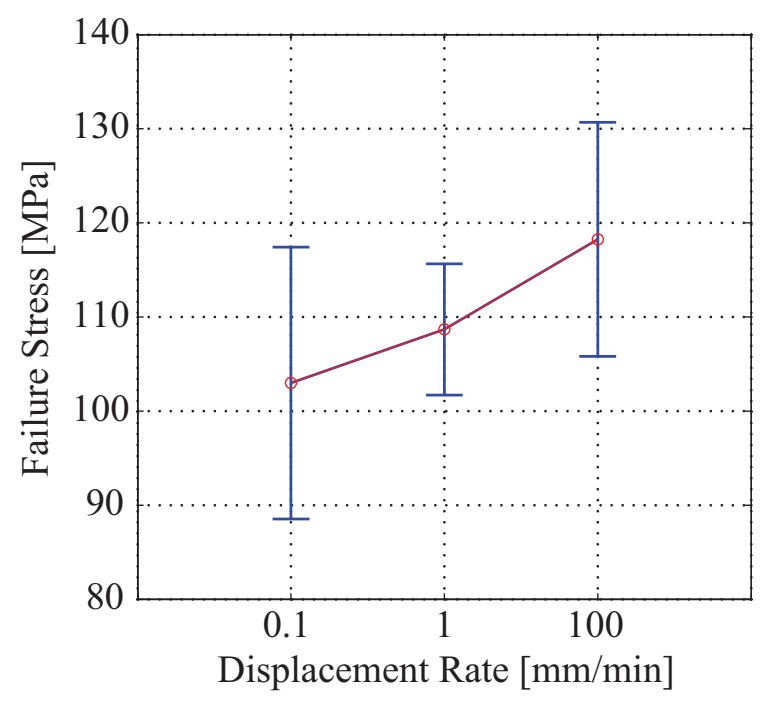

Figure 8: Effect of loading rate on failure stress in three point bending specimens.

simulations, the standard deviations are 7.08, 6.62, and $8.37 \mathrm{MPa}$, respectively. The percent errors between predicted and experimental standard deviations are $7.4 \%, 5.0 \%$, and $32.7 \%$, respectively. The variance in the $0.1 \mathrm{~mm} / \mathrm{min}$ and $1 \mathrm{~mm} / \mathrm{min}$ displacement rates are in good agreement between the experiments and simulations. The experimental variance for the fastest displacement rate was much higher than for the slower two rates. This displacement rate was near the limits of the capabilities of the MTS testing machine. At the high rate of loading, the greater variance compared to the variance from the other experiments could be attributed to a greater degree of measurement error in the fastest displacement rate, as well as the presence of additional physical phenomena that are significant only at the highest loading rate. The standard deviation from the simulations performed at the fastest displacement rate was much closer to the simulations of the other two rates than was witnessed in the corresponding experiments.

The PDFs obtained through multiscale simulations with calibrated parameter distributions show some discrepancy with those obtained by the experiments. The primary reason for the discrepancies is the modeling error associated with the idealization of rate effects. The calibration of matrix material properties were performed to match behavior at three different load rates together. When parameters are allowed to vary from rate to rate (i.e., calibration performed using experiments performed at a single rate), the experimental and simulated PDFs match to the same degree as Fig. 6. This result implies a better model is necessary to capture the rate effect compared to the power law used here. Nevertheless, a good match is observed between the primary distribution metrics (i.e. mean and variance). 

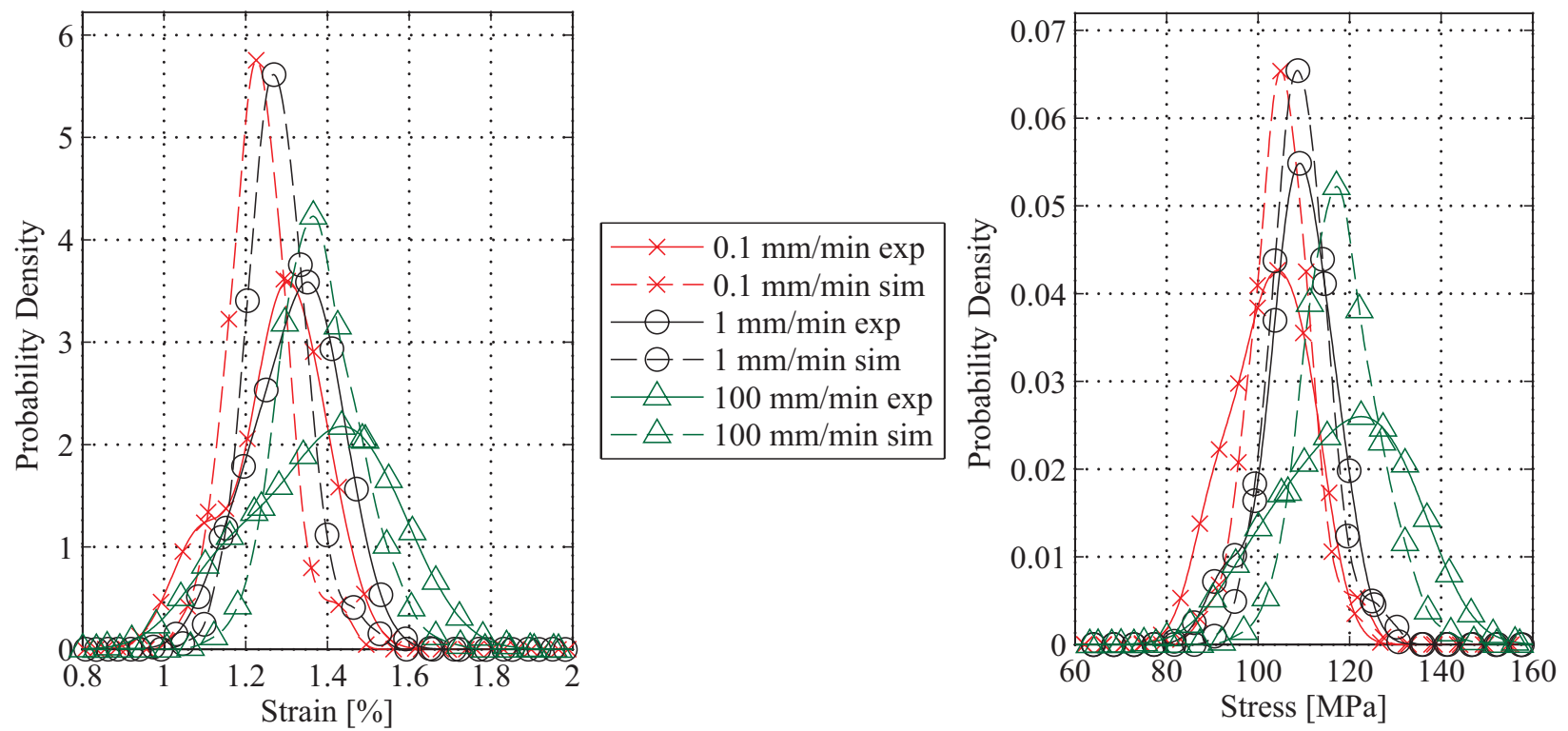

Figure 9: KS density estimations of PDFs of strain to failure and ultimate stress for experimental results and simulated samples from calibrated parameter distributions for $90^{\circ}$ monotonic three point bend tests.

Table 5: Experimental and calibrated failure properties of unidirectional $90^{\circ}$ specimens.

\begin{tabular}{ccccccc}
\hline & & \multicolumn{2}{c}{ Experiments } & & \multicolumn{2}{c}{ Calibration } \\
\cline { 3 - 4 } \cline { 6 - 7 } Property & Rate & Mean & CoV & & Mean & CoV \\
\hline$X_{f}^{2}[\mathrm{MPa}]$ & $0.1 \mathrm{~mm} / \mathrm{min}$ & 102.3 & 0.075 & & 104.5 & 0.068 \\
& $1 \mathrm{~mm} / \mathrm{min}$ & 108.7 & 0.064 & & 108.7 & 0.061 \\
& $100 \mathrm{~mm} / \mathrm{min}$ & 118.3 & 0.105 & & 117.9 & 0.071 \\
$\epsilon_{f}^{2}[\%]$ & $0.1 \mathrm{~mm} / \mathrm{min}$ & 1.27 & 0.084 & & 1.22 & 0.064 \\
& $1 \mathrm{~mm} / \mathrm{min}$ & 1.32 & 0.073 & & 1.27 & 0.060 \\
& $100 \mathrm{~mm} / \mathrm{min}$ & 1.38 & 0.112 & & 1.38 & 0.075 \\
\hline
\end{tabular}

\subsection{Model Validation}

The calibrated probabilistic multiscale model was employed to predict the failure response of openhole quasi-isotropic $[+45,0,-45,90]_{2 s}$ specimens subjected to uniaxial tension at two different strain rates and validated against the observed experimental data. Twenty random correlated parameter sets were drawn from the calibrated parameter distributions. These parameters were used in multiscale simulations with the finite element discretization shown in Fig. 10. A structured mesh was used in each ply of the quasi-isotropic layup such that the elements were oriented along the fiber direction to minimize mesh bias, an approach described in Ref. [36]. The surface meshes between 
two neighboring plies were therefore not necessarily identical. The inter-ply continuity was imposed by employing node-to-surface constraints. We note that delamination, which is sometimes modeled using cohesive elements along ply interfaces, is modeled at the microscale within the sEHM framework. Inclusion of cohesive elements is therefore not needed.

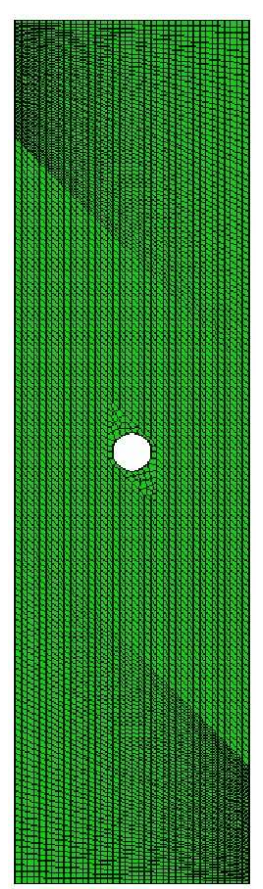

(a) $\pm 45^{\circ}$

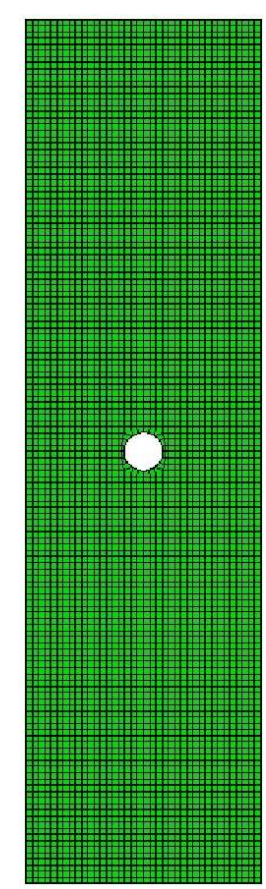

(b) $0 / 90^{\circ}$

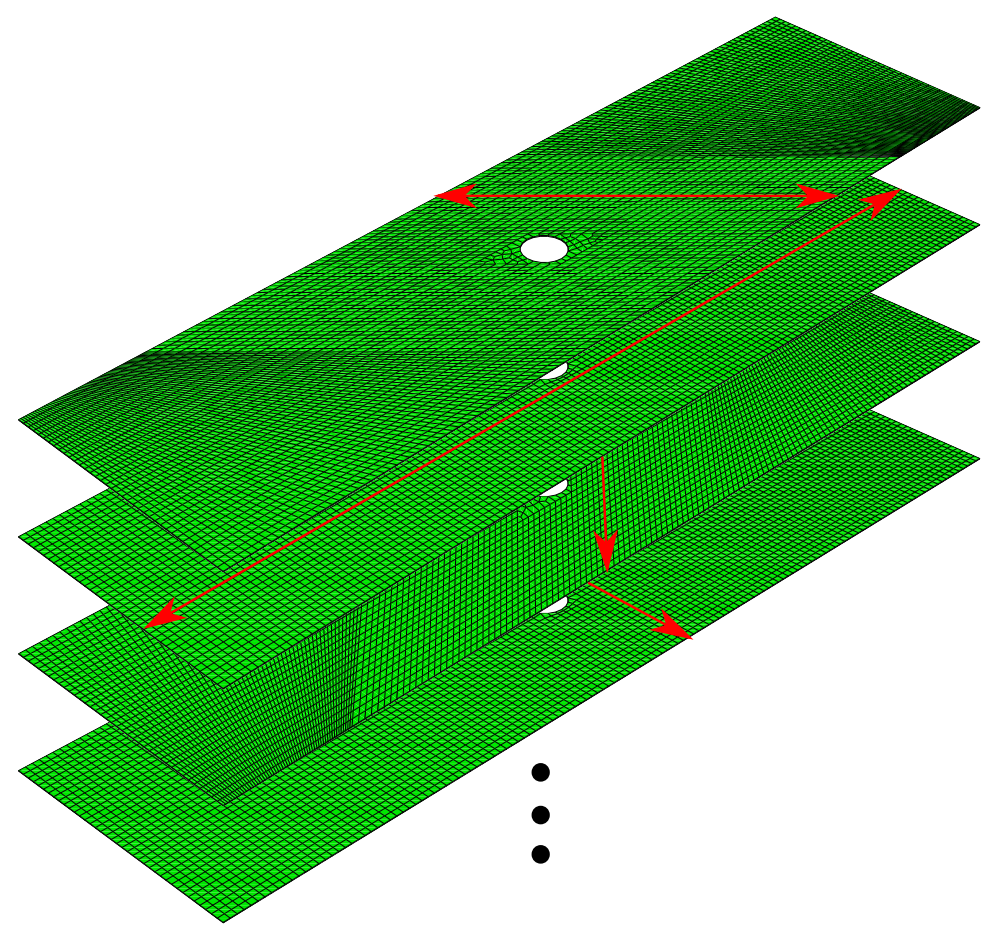

(c) Laminated Composite

Figure 10: Fiber aligned mesh for (a) \pm 450 plies, (b) $0 / 90^{\circ}$ plies, and (c) $[+45,0,-45,90]_{2 s}$ laminated composite.

The simulated stress strain curves at the loading rates of $1 \mathrm{~mm} / \mathrm{min}$ and $100 \mathrm{~mm} / \mathrm{min}$ are shown against the experiments in Fig. 11. The average failure stresses from the experiments were 471.2 and $486.8 \mathrm{MPa}$ at the $1 \mathrm{~mm} / \mathrm{min}$ and $100 \mathrm{~mm} / \mathrm{min}$ loading rates, respectively. A consistent failure mechanism was observed in each of the open hole specimens, predominated by transverse matrix cracking at the hole in the $90^{\circ}$ and $\pm 45^{\circ}$ plies and fiber failure in the $0^{\circ}$ plies, as seen in Fig. 12. The corresponding average failure stresses predicted by the simulations was $476.5 \mathrm{MPa}$ and 485.1 MPa. The predictions of the mean strength are in excellent agreement with the experimental observations. The standard deviation of failure stress from the experiments was $20.4 \mathrm{MPa}$ and 31.2 MPa for the slow and fast loading rates, respectively, compared to $30.5 \mathrm{MPa}$ and 34.1 MPa, respectively, from the calibrated predictions. It is noted that only three experiments were available for each loading rate and more comprehensive experimental datasets are needed to fully validate the proposed approach with regards to the standard deviation of the predictions.

The simulations shown in Fig. 11 considered the failure parameters of the composite constituents 


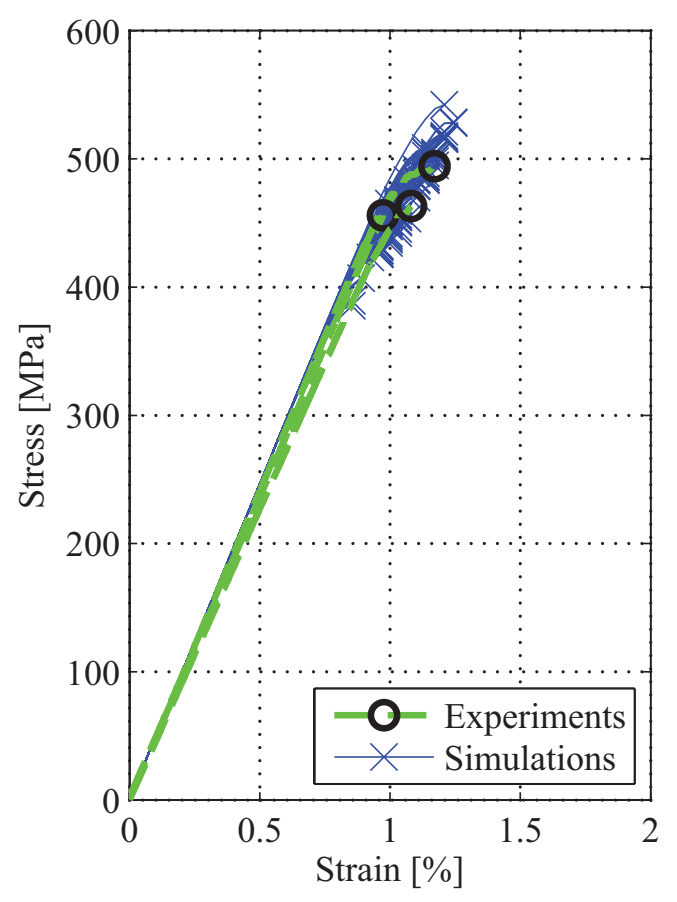

(a)

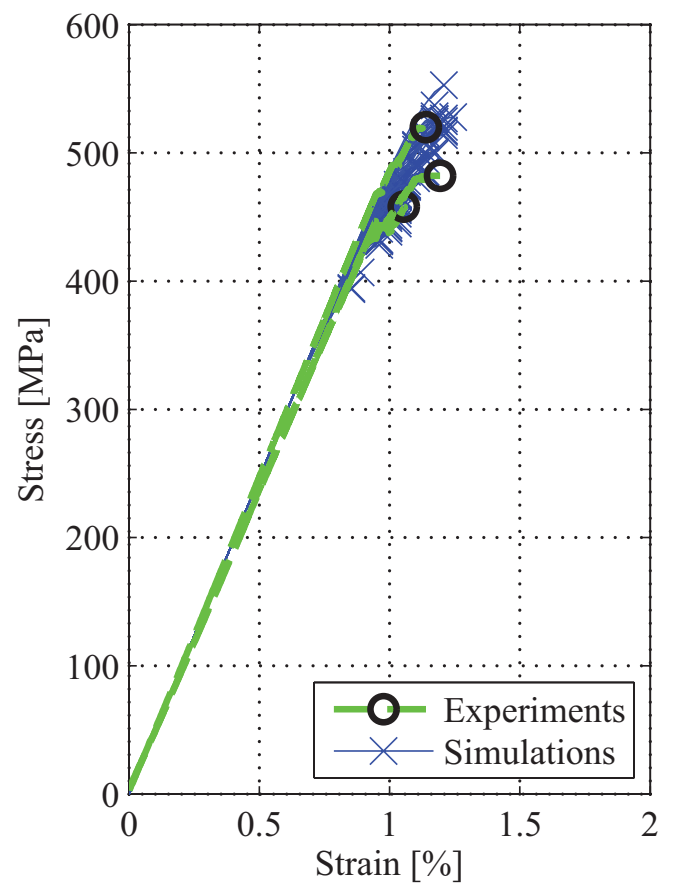

(b)

Figure 11: Stress-strain curves for experiments and simulations of quasi-isotropic open hole specimens in monotonic tension at (a) $1 \mathrm{~mm} / \mathrm{min}$ and (b) $100 \mathrm{~mm} / \mathrm{min}$.

as randomly sampled from the calibrated distributions, but spatially uniform across the specimen. An additional investigation was performed to understand the effects of treating the material parameters as spatially varying over the specimen. We introduce the length scale parameter, $l_{r}$, such that a structure with smaller $l_{r}$ indicates more rapidly fluctuating parameter values across the specimen realization than one with a larger $l_{r}$. In this investigation, the random parameters were sampled for the structure in groups parallel to the fiber orientation of width equal to $l_{r}$, as shown in Fig. 13. This sampling method was selected as fiber properties were taken to be constant along the length of the fiber and the in situ matrix properties to be strongly dependent on the confining effects of fiber spacing, which was also taken to be constant along the length of the fibers.

The samples were drawn from the distributions determined from the MCMC calibration procedure. For each length scale parameter considered, 20 random spatially varying parameter fields were generated and the multiscale model was exercised to predict the ultimate failure for each realization. The resulting mean and standard deviation of the ultimate failure strength in the quasi-isotropic open hole coupon under tension at the slow loading rate is presented in Fig. 14. The sampling width does not have a significant impact on the mean failure strength of the coupon. However, there is a pronounced effect on the standard deviation of predicted failures. For realizations with a high sampling length, i.e. the parameters fluctuate at a lower frequency over the realization, the standard 

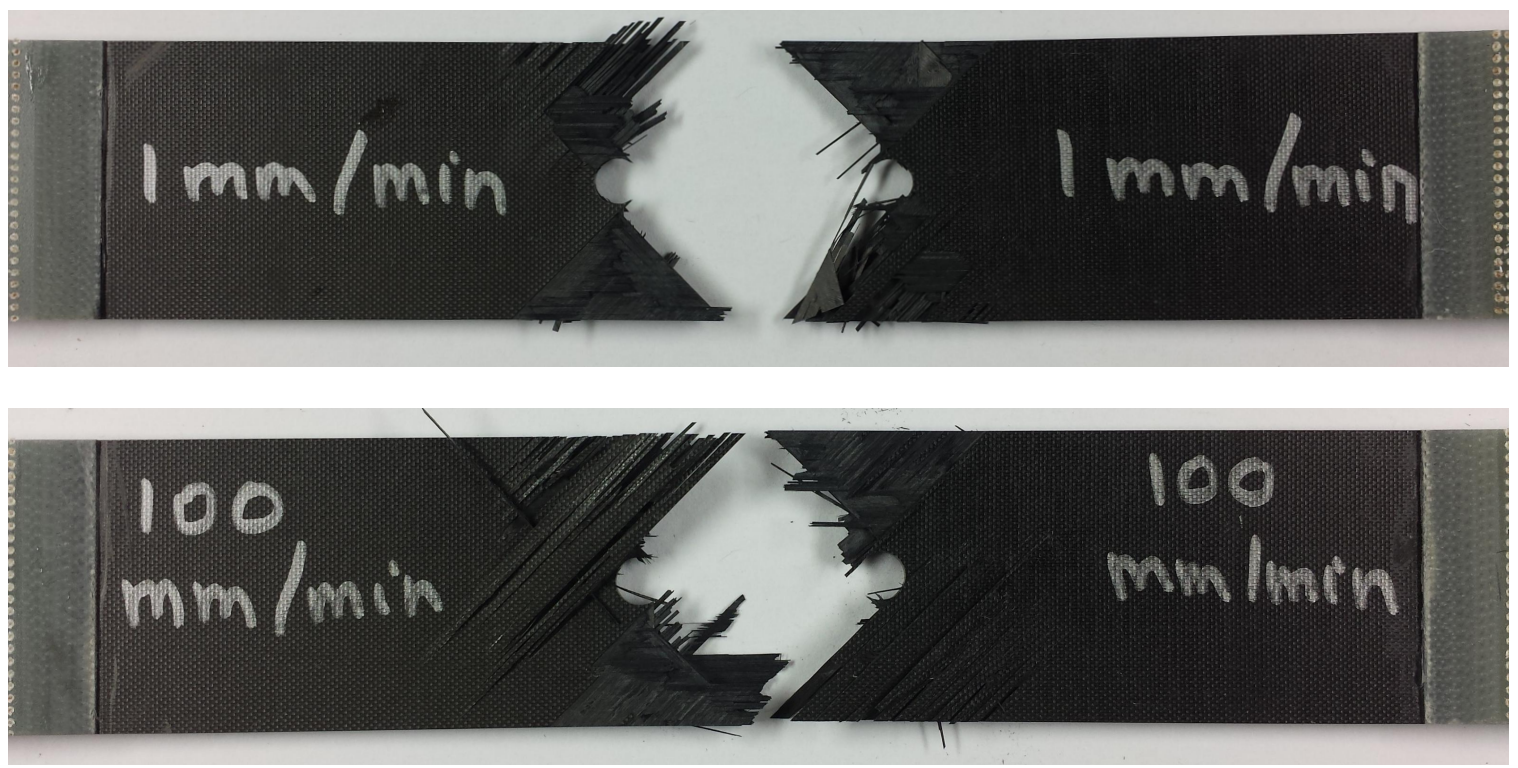

Figure 12: Images of failure from $[+45,0,-45,90]_{2 s}$ quasi-isotropic open hole tension tests at 1.0 $\mathrm{mm} / \mathrm{min}$ (top figure) and $100 \mathrm{~mm} / \mathrm{min}$ (bottom figure).

deviation is larger than the realizations with more rapidly fluctuating parameters. A logarithmic fit line for the standard deviation as a function of sampling length is included in Fig. 14(b).

\section{Conclusion}

This manuscript presented a multiscale modeling approach to predict the failure behavior of composite materials under uncertainty. The Bayesian framework is the foundation of the proposed multiscale uncertainty quantification approach. The proposed approach was demonstrated to successfully predict the mean experimental failure observed in fiber reinforced laminated polymer composites subjected to static loading at various load rates, as well as capture the variability in this behavior. A key capability of this approach is that uncertainty can be propagated not only from the constituent scale to the laminate level, but also from the laminate level to the constituent scale. This is done in the context of the reduced order computational homogenization model.

While the proposed approach is capable of quantifying uncertainty in composites and upscaling variability, several challenges remain to be resolved. First is addressing the presence of several sources of uncertainty at the scale of the microstructure. Composite microstructures have many topological uncertainties (e.g., randomness of fiber orientation, volume fraction, void distribution) which are only implicitly accounted for in the current formulation. Incorporation of topological variabilities and distinguishing them from the parameter variability remains outstanding. In this study, the coefficients of variation in both calibration and validation experiments were between 6 and $12 \%$. While the proposed calibration procedure does not depend on the magnitude of varia- 


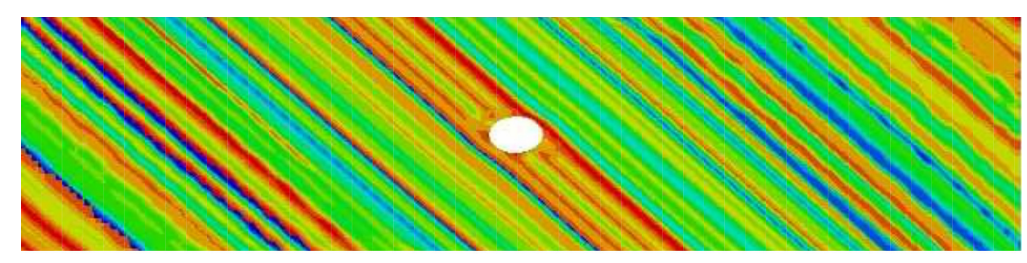

(a)

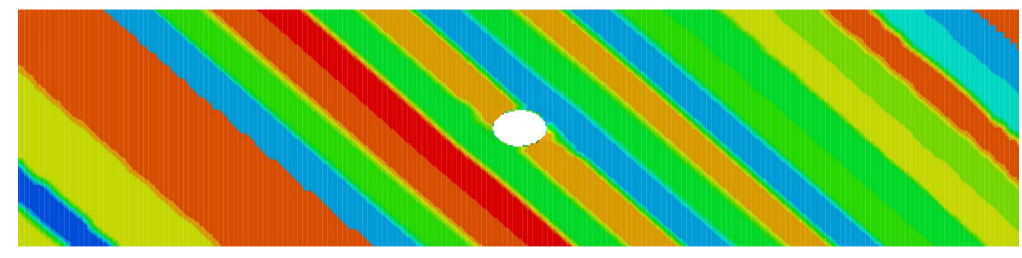

(b)

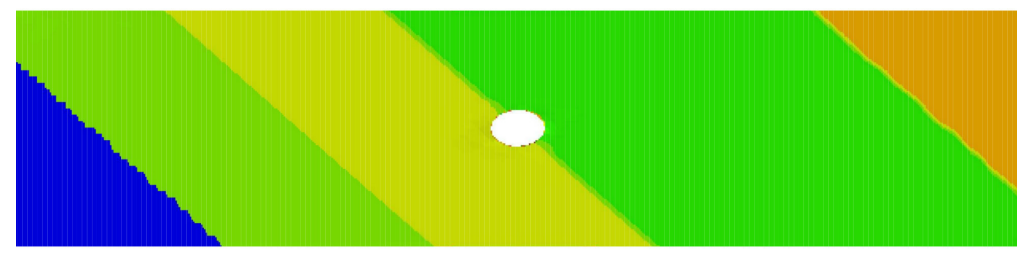

(c)

Figure 13: Sampled parameter groups for $l_{r}=$ (a) $1 \mathrm{~mm}$, (b) $5 \mathrm{~mm}$, (c) $20 \mathrm{~mm}$

tion, magnitudes may be exacerbated by the presence of additional sources of uncertainty in other scenarios. Larger variances would also require a higher number of realizations to sample the larger parameter space in numerical predictions, and may cause an increase in the error of predicted failure properties. A second challenge is capturing the tails of the distributions. The reliability-based composite design process relies on accurately capturing very low levels of failure probability of a composite component. The multiscale uncertainty quantification approach must therefore accurately represent the tails of the distributions. A comprehensive experimental-computational study is needed to validate the performance of the proposed approach in capturing the distributions at such low levels of failure probability.

\section{Acknowledgements}

The authors gratefully acknowledge the financial support provided by the Aerospace Systems Directorate of the Air Force Research Laboratory (Contract No: GS04T09DBC0017 through Engility Corporation) and the Department of Defense (DoD) through the National Defense Science \& Engineering Graduate Fellowship (NDSEG) Program. 


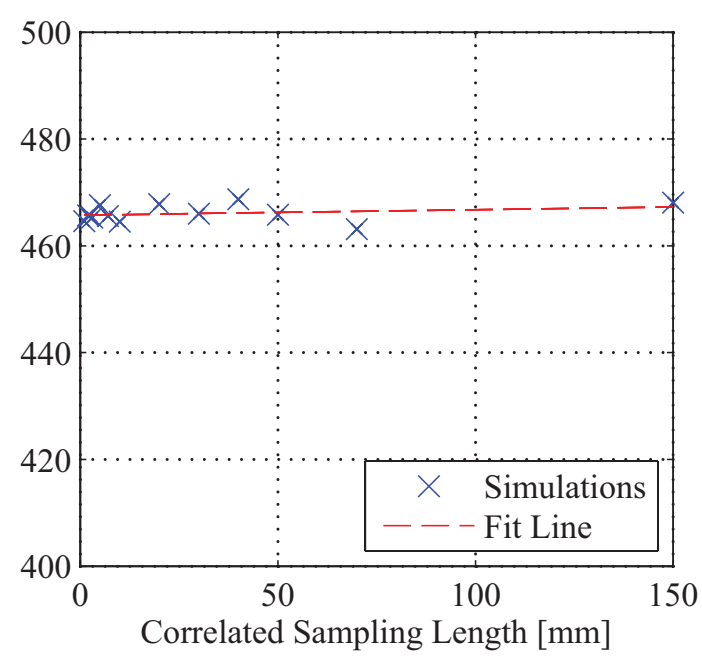

(a)

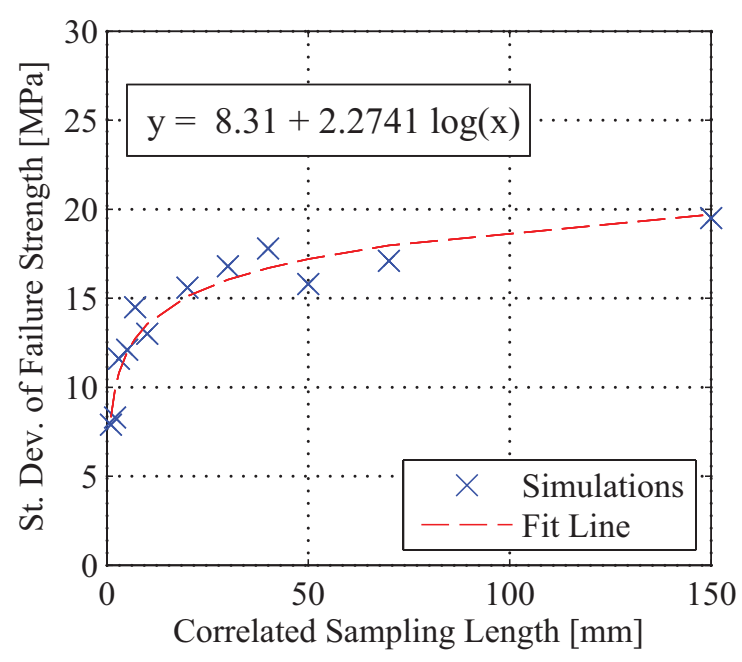

(b)

Figure 14: (a) Mean and (b) standard deviation of ultimate failure strength as a function of the length of correlated sampling.

\section{References}

[1] ASTM Standard D3039. Standard test method for tensile properties of polymer matrix composite materials. ASTM International, West Conshohocken, PA, 2008.

[2] ASTM Standard D5766. Standard test method for open-hole tensile strength of polymer matrix composite laminates. ASTM International, West Conshohocken, PA, 2011.

[3] ASTM Standard D790. Standard test methods for flexural properties of unreinforced and reinforced plastics and electrical insulating materials. ASTM International, West Conshohocken, PA, 2010.

[4] M. J. Bogdanor, S. Mahadevan, and C. Oskay. Uncertainty quantification in damage modeling of heterogeneous materials. Int. J. Multiscale Com., 11:287-307, 2013.

[5] C. C. Chamis. Probabilistic simulation of multi-scale composite behavior. Theor. Appl. Fract. Mec., 41:51-61, 2004.

[6] N. Z. Chen and C. G. Soares. Spectral stochastic finite element analysis for laminated composite plates. Comput. Meth. Appl. Mech. Engrg., 197:4830-4839, 2008.

[7] A. Clement, C. Soize, and J. Yvonnet. Uncertainty quantification in computational stochastic multiscale analysis of nonlinear elastic materials. Comput. Meth. Appl. Mech. Engrg., pages 61-82, 2013. 
[8] R. Crouch and C. Oskay. Symmetric mesomechanical model for failure analysis of heterogeneous materials. Int. J. Multiscale Com., 8:447-461, 2010.

[9] R. Crouch and C. Oskay. Experimental and computational investigation of progressive damage accumulation in CFRP composites. Compos. Part B-Eng., 48:59-67, 2013.

[10] R. Crouch, C. Oskay, and S. Clay. Multiscale modeling of damage accumulation in carbon fiber reinforced polymers subjected to fatigue. Proceedings of the 53rd AIAA Structures, Structural Dynamics, and Materials Conference, 2012.

[11] R. Crouch, C. Oskay, and S. Clay. Multiple spatio-temporal scale modeling of composites subjected to cyclic loading. Comput. Mech., 51:93-107, 2013.

[12] M. M. S. Dwaikat, C. Spitas, and V. Spitas. Effect of the stochastic nature of the constituents parameters on the predictability of the elastic properties of fibrous nano-composites. Compos. Sci. Technol., 2012.

[13] J. Fish and W. Wu. A nonintrusive stochastic multiscale solver. Int. J. Numer. Meth. Engng., 88:862-879, 2011.

[14] J. Fish and Q. Yu. Multiscale damage modelling for composite materials: Theory and computational framework. Int. J. Numer. Meth. Engng., 52:161-191, 2001.

[15] T. Gerstner and M. Griebel. Numerical integration using sparse grids. Numer. Algorithms, 18:209-232, 1998.

[16] M. S. Greene, H. Xu, S. Tang, W. Chen, and W. K. Liu. A generalized uncertainty propagation criterion from benchmark studies of microstructured material systems. Comput. Meth. Appl. Mech. Engrg., 254:271-291, 2013.

[17] J. M. Guedes and N. Kikuchi. Preprocessing and postprocessing for materials based on the homogenization method with adaptive finite element methods. Comput. Meth. Appl. Mech. Engrg., 83:143-198, 1990.

[18] V. Hombal and S. Mahadevan. Bias minimization in gaussian process surrogate modeling for uncertainty quantification. Int. J. Uncertain. Quantif., 1:321-349, 2011.

[19] T. Hui and C. Oskay. Computational modeling of polyurea-coated composites subjected to blast loads. J. Compos. Mater., 46:2167-2178, 2012.

[20] M. Kamiński and M. Kleiber. Perturbation based stochastic finite element method for homogenization of two-phase elastic composites. Comput. Struct., 78:811-826, 2000. 
[21] D.J. Lekou and T.P. Philippidis. Mechanical property variability in FRP laminates and its effect on failure prediction. Compos. Part B-Eng., 39:1247-1256, 2008.

[22] S. C. Lin. Reliability predictions of laminated composite plates with random system parameters. Probabilist. Eng. Mech., 15:327-338, 2000.

[23] P.A.M. Lopes, H.M. Gomes, and A.M. Awruch. Reliability analysis of laminated composite structures using finite elements and neural networks. Compos. Struct., 92:1603-1613, 2010.

[24] C. Oskay and J. Fish. Eigendeformation-based reduced order homogenization for failure analysis of heterogeneous materials. Comput. Method. Appl. Mech. Engrg., 196:1216-1243, 2007.

[25] E. Parzen. On estimation of a probability density function and mode. Ann. Math. Stat., 33:1065-1076, 1962.

[26] C. Rasmussen and C. Williams. Gaussian Processes for Machine Learning. Springer, New York, 2006.

[27] M. Rosenblatt. Remarks on some nonparametric estimates of a density function. The Annals of Mathematical Statistics, 27:832-837, 1956.

[28] S. Sakata, F. Ashida, and K. Enya. Stochastic analysis of microscopic stress in fiber reinforced composites considering uncertainty in a microscopic elastic property. J. Solid Mech. Mater. Eng., 4:568-577, 2010.

[29] S. Sakata, F. Ashida, T. Kojima, and M. Zako. Three-dimensional stochastic analysis using a perturbation-based homogenization method for elastic properties of composite material considering microscopic uncertainty. Int. J. Solids Struct., 45(3):894-907, 2008.

[30] S. Sakata, F. Ashida, and M. Zako. Kriging-based approximate stochastic homogenization analysis for composite materials. Comput. Method. Appl. M., 197:1953-1964, 2008.

[31] S. Sankararaman, Y. Ling, and S. Mahadevan. Uncertainty quantification and model validation of fatigue crack growth prediction. Eng. Fract. Mech., 78:1487-1504, 2011.

[32] A. Shaw, S. Sriramula, P. D. Gosling, and M. K. Chryssanthopoulos. A critical reliability evaluation of fibre reinforced composite materials based on probabilistic micro and macromechanical analysis. Compos. Part B-Eng., 41:446-453, 2010.

[33] M. C. Shiao and C. C. Chamis. Probabilistic evaluation of fuselage-type composite structures. Probabilist. Eng. Mech., 14:179-187, 1999.

[34] B. W. Silverman. Density estimation for statistics and data analysis, volume 26. CRC press, 1986. 
[35] J.C. Simo and J.W. Ju. Strain- and stress-based continuum damage models - I. Formulation. Int. J. Solids. Struct., 23:821-840, 1987.

[36] K. Song, Y. Li, and C. A. Rose. Continuum damage mechanics models for the analysis of progressive failure in open-hole tension laminates. Proceedings of the 52nd AIAA Structures, Structural Dynamics, and Materials Conference, 1861:1-18, 2011.

[37] S. Sriramula and M. K. Chryssanthopoulos. Quantification of uncertainty modelling in stochastic analysis of FRP composites. Compos. Part A-Appl. S., 40:1673-1684, 2009.

[38] M. Tootkaboni and L. Graham-Brady. A multi-scale spectral stochastic method for homogenization of multi-phase periodic composites with random material properties. Int. J. Numer. Meth. Eng., 83:59-90, 2010.

[39] D. Xiu and G. E. Karniadakis. The wiener-askey polynomial chaos for stochastic differential equations. SIAM J. Sci. Comput., 24:619-644, 2002.

[40] H. Yan, C. Oskay, A. Krishnan, and L. R. Xu. Compression after impact response of woven fiber reinforced composites. Compos. Sci. Technol., 70:2128-2136, 2010.

[41] J. Yvonnet, E. Monteiro, and Q.-C. He. Computational homogenization method and reduced database model for hyperelastic heterogeneous structures. Int. J. Mult. Comp. Eng., 11:201$225,2013$. 